\title{
XUV Photometer System (XPS): Improved Solar Irradiance Algorithm Using CHIANTI Spectral Models
}

\author{
Thomas N. Woods • Phillip C. Chamberlin • W.K. Peterson • R.R. Meier • \\ Phil G. Richards • Douglas J. Strickland • Gang Lu • Liying Qian • \\ Stanley C. Solomon · B.A. Iijima • A.J. Mannucci · B.T. Tsurutani
}

Received: 26 December 2007 / Accepted: 22 April 2008 / Published online: 30 May 2008

(C) The Author(s) 2008

\begin{abstract}
Solar soft X-ray (XUV) radiation is highly variable on all time scales and strongly affects Earth's ionosphere and upper atmosphere; consequently, the solar XUV irradiance is
\end{abstract}

T.N. Woods $(\bowtie) \cdot$ P.C. Chamberlin · W.K. Peterson

Laboratory for Atmospheric and Space Physics, University of Colorado, Boulder, CO 80303, USA

e-mail: tom.woods@lasp.colorado.edu

P.C. Chamberlin

e-mail: phil.chamberlin@lasp.colorado.edu

W.K. Peterson

e-mail: bill.peterson@lasp.colorado.edu

R.R. Meier · P.G. Richards

Physics and Astronomy Department, George Mason University, Fairfax, VA 22030, USA

R.R. Meier

e-mail: rmeier@gmu.edu

P.G. Richards

e-mail: prichar1@gmu.edu

D.J. Strickland

Computational Physics, Inc., Springfield, VA 22151, USA

e-mail: dstrick@cpi.com

G. Lu · L. Qian · S.C. Solomon

High Altitude Observatory, National Center for Atmospheric Research, Boulder, CO 80309, USA

G. Lu

e-mail: gang.lu@hao.ucar.edu

L. Qian

e-mail: Iqian@ucar.edu

S.C. Solomon

e-mail: stans@ucar.edu

B.A. Iijima · A.J. Mannucci · B.T. Tsurutani

Jet Propulsion Laboratory, Pasadena, CA 91109, USA 
important for atmospheric studies and for space weather applications. Although there have been several recent measurements of the solar XUV irradiance, detailed understanding of the solar XUV irradiance, especially its variability during flares, has been hampered by the broad bands measured in the XUV range. In particular, the simple conversion of the XUV photometer signal into irradiance, in which a static solar spectrum is assumed, overestimates the flare variations by more than a factor of two as compared to the atmospheric response to the flares. To address this deficiency in the simple conversion, an improved algorithm using CHIANTI spectral models has been developed to process the XUV Photometer System (XPS) measurements with its broadband photometers. Model spectra representative of quiet Sun, active region, and flares are combined to match the signals from the XPS and produce spectra from 0.1 to $40 \mathrm{~nm}$ in $0.1-\mathrm{nm}$ intervals for the XPS Level 4 data product. The two XPS instruments are aboard NASA's Solar Radiation and Climate Experiment (SORCE) and Thermosphere, Ionosphere, Mesosphere, Energetics, and Dynamics (TIMED) satellites. In addition, the XPS responsivities have been updated for the latest XPS data processing version. The new XPS results are consistent with daily variations from the previous simple conversion technique used for XPS and are also consistent with spectral measurements made at wavelengths longer than $27 \mathrm{~nm}$. Most importantly, the XPS flare variations are reduced by factors of 2-4 at wavelengths shorter than $14 \mathrm{~nm}$ and are more consistent, for the first time, with atmospheric response to solar flares. Along with the details of the new XPS algorithm, several comparisons to dayglow and photoelectron measurements and model results are also presented to help verify the accuracy of the new XUV irradiance spectra.

\section{Introduction}

Solar soft X-ray (XUV) radiation is highly variable on all time scales with variations on both short-term (on the order of minutes for flares) and long-term (on the order of years for the solar cycle) scales, ranging from (wavelength-dependent) factors of 2 to 100 . These solar XUV variations directly affect the composition, density, and temperature in Earth's ionosphere and upper atmosphere. Furthermore, the solar-driven atmospheric processes are wavelength dependent and thus dependent on the intrinsic solar variability at the appropriate wavelengths. Large flares are of major concern for space weather applications such as their effects on communication and navigation resulting from ionospheric changes (e.g., Lanzerotti, 2001; Kintner, Ledvina, and de Paula, 2007) and on satellite tracking as related to satellite drag changes from solar forcing of the neutral density (e.g., Sutton et al., 2006). An example of the flare variation is shown in Figure 1 for the X28 flare on 4 November 2003, the largest flare observed since the routine monitoring of solar X rays commenced in the 1970s. In addition, solar cycle changes are important for understanding the long-term changes in the upper atmosphere such as the decrease in thermospheric neutral density (e.g., Marcos et al., 2005; Qian et al., 2006). Accurate measurements of the solar ultraviolet spectral irradiance, along with an understanding of its variability on all time scales, are required for atmospheric studies and application for space weather operations.

B.A. Iijima

e-mail: byron.a.iijima@ @pl.nasa.gov

A.J. Mannucci

e-mail: anthony.j.mannucci@jpl.nasa.gov

B.T. Tsurutani

e-mail: bruce.t.tsurutani@jpl.nasa.gov 

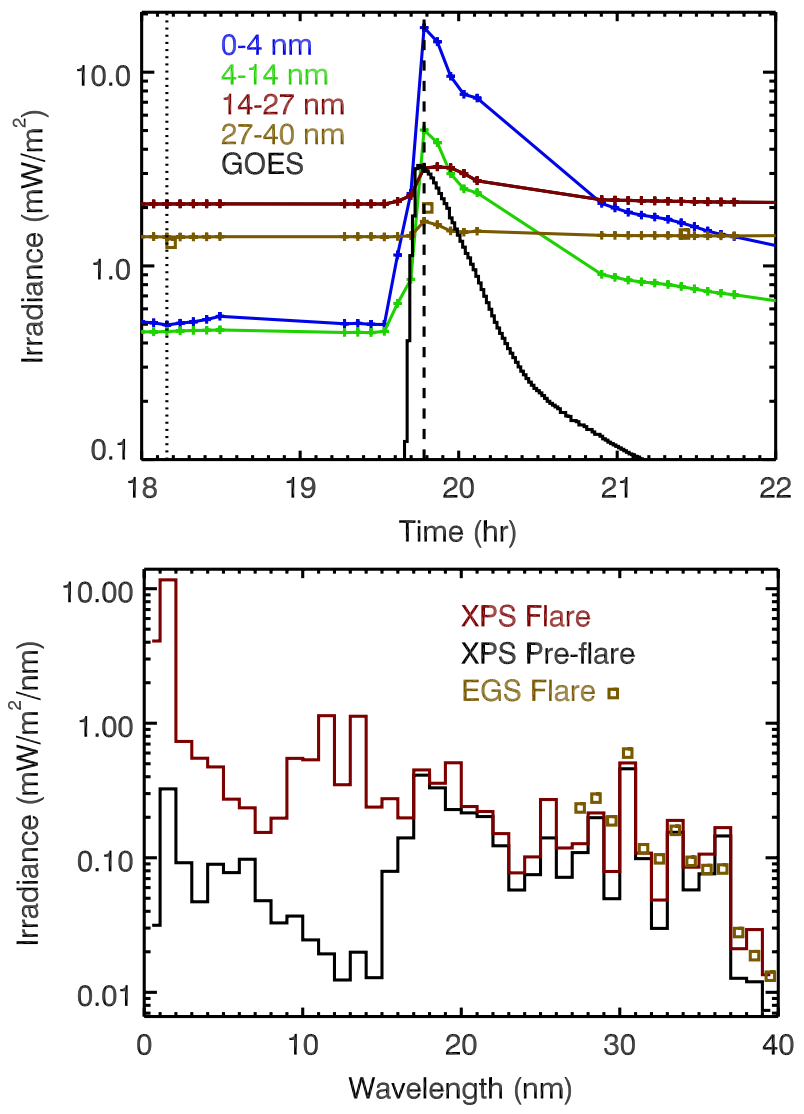

Figure 1 Example flare time series and spectra. In the top panel, the time series of the large X28 flare on 4 November 2003 is shown for several bands from the new XPS Level 4 product, along with the GOES $0.1-0.8 \mathrm{~nm}$ X-ray measurement. As for this example, the $0-4 \mathrm{~nm}$ band is often the dominant emission during a flare but is a minor contribution at other times. In the bottom panel, the spectrum near the peak of the flare (dashed line in top panel) is compared to the pre-flare spectrum (dotted line in top panel). The majority of the irradiance increase is shorter than $15 \mathrm{~nm}$. The XPS Level 4 model results are in reasonably good agreement with the SEE EGS spectral measurements at longer than $27 \mathrm{~nm}$.

Studies of the solar XUV radiation began in the 1950s with space-based rocket experiments, but our knowledge of the solar XUV irradiance, both in absolute magnitude and variability, has been incomplete largely because of the very limited number of observations and because the measurements are mainly from broadband photometers. Nominal subdivisions of the short ultraviolet spectral range are the extreme ultraviolet (EUV) from 30 to $120 \mathrm{~nm}, \mathrm{X}$-ray ultraviolet (XUV) from 1 and $30 \mathrm{~nm}$, and X rays at wavelengths less than $1 \mathrm{~nm}$. For discussion here, the "XUV" measurements from the XUV Photometer System (XPS) are in the 0.1-40 nm range. With the launch of the Solar and Heliospheric Observatory (SOHO) in December 1995, Student Nitric Oxide Explorer (SNOE) in February 1998, Thermosphere, Ionosphere, Mesosphere, Energetics, and Dynamics (TIMED) spacecraft in December 2001, and Solar Radiation and Climate Experiment (SORCE) spacecraft in January 2003, there is now a continuous data set of the solar XUV irradiance, advancing our understanding of the solar XUV irradiance. Recent reviews of the solar EUV and UV 
variability with more details include those by White (1977), Rottman (1987), Lean (1987, 1991), Tobiska (1993), Pap et al. (1994), and Woods et al. (2004a). Although there have been several recent measurements of the solar XUV irradiance, detailed understanding of the solar XUV irradiance, especially its variability during flares, has been hampered by the broad bands measured in the XUV range.

The spectral variations during flares are of acute interest for space weather research and applications. There is, on average, one modest M-class solar flare every two days and one large X-class flare per month as determined from a survey of flares during solar cycles 21 and 22 (Garcia, 2000). However, it is important to note that there are more flares during solar maximum conditions than at solar minimum. Although flares are observable at all wavelengths, most flares affect the solar irradiance primarily in the X-ray region, but sometimes a large flare can affect the solar irradiance over a broad wavelength range (e.g., Brekke et al., 1996; Meier et al., 2002; Woods et al., 2003). The simultaneous spectral measurements over the 27-193 $\mathrm{nm}$ range by the TIMED Solar EUV Experiment (SEE), although limited to only some of the flares because of its $3 \%$ duty cycle, have advanced our understanding of variations during both the impulsive and gradual phases of flares (e.g., Woods et al., 2003, 2004b, 2005, Woods, Kopp, and Chamberlin, 2006). Consequently, new models of the flare variations, such as the Flare Irradiance Spectral Model (FISM), are now being developed (Chamberlin, Woods, and Eparvier, 2007).

Although the flare variations are better understood at longer than $27 \mathrm{~nm}$, the results with the broadband measurements at shorter than $27 \mathrm{~nm}$ initially yielded conflicting results with atmospheric responses to flares (e.g., Strickland et al., 2007). In particular, the simple conversion of the XUV photometer signal into irradiance, in which a static solar spectrum is assumed, overestimates the flare variations by more than a factor of 2 as compared to the atmospheric response to the flares. A new algorithm for processing the XPS data, which is the focus of this paper, uses dynamic solar spectra. Consequently, the flare variations are reduced because the spectral shape changes for each measurement, and better agreement is now found with the observed atmospheric response to flares. In addition, the XPS responsivities have been updated for the latest XPS data-processing version.

This improved XPS processing algorithm uses CHIANTI spectral models (Dere et al., 1997; Landi et al., 2006) that are scaled to match the signals from the broadband XUV photometers. Model spectra representative of quiet Sun, active region, and flares are combined to match the signals from the XPS and produce spectra from 0.1 to $40 \mathrm{~nm}$ in $0.1-\mathrm{nm}$ intervals for the XPS Level 4 data product. The primary improvement from the previous XPS data version (which only used a single solar spectrum for each day) is that the GOES X-ray irradiance measurements are used in this new algorithm to provide an estimate of the flare temperature for use in the CHIANTI spectral model for each XPS measurement. This new algorithm and results for the new XPS data products are the focus for this paper, along with selected comparisons to the atmospheric response to a few flares.

\section{XPS Instrument Overview}

The XPS comprises a set of filter photometers that measure the solar irradiance from 0.1 to $27 \mathrm{~nm}$ with an additional channel at the important $\mathrm{H}$ I Lyman- $\alpha$ line at $121.6 \mathrm{~nm}$. The XPS is one of four different solar irradiance instruments on NASA's SORCE satellite (Rottman, 2005; Woods, Rottman, and Vest, 2005) and is also part of SEE on NASA's TIMED satellite (Woods et al., 2005). In each XPS there are a total of twelve silicon photodiodes, eight with metal films directly deposited on them, one with 121-nm interference filters in front, and 
three with bare photodiodes. The filter material (either metal coating or interference) establishes the wavelength sensitivity over broad bands of about 7-10 $\mathrm{nm}$ and also blocks the long-wavelength solar radiation that would overwhelm the relatively weak signal at these short X-ray wavelengths. The twelve photometers are packaged in a single unit with a filter wheel mechanism in front. As the wheel turns, it places an open aperture, a blocked position, or a window (of fused silica) in front of each diode. For the nine filter diodes the open aperture allows the solar irradiance measurement, the blocked position provides a reading of the photodiode background (dark) signal, and the window position measures the long-wavelength (visible) leakage through the filter. The three bare diodes provide in-flight calibrations of the window transmission.

The primary data products from XPS are the solar XUV irradiance in its broad bands and also at higher spectral resolution based on scaling model spectra to the XPS signal levels. The time cadences for the XPS results include the daily averaged irradiances and higher cadence of a few minutes. Because of the satellite orbit and instrument configuration, the TIMED XPS has about a 3\% duty cycle for solar observations, and the SORCE XPS has about a $70 \%$ duty cycle for solar observations. The TIMED daily measurements began in January 2002, and the SORCE daily measurements began in March 2003. Both continue to the present with very few data gaps. A data gap in each XPS solar record is related to an anomaly with its filter wheel mechanism. On 24 July 2002, the TIMED XPS filter mechanism became stuck in position 6 and has remained in that position. From this position, the TIMED XPS has three photometers making valid XUV measurements, most notably the one with the Ti filter, which makes the primary measurement in the $0.1-7 \mathrm{~nm}$ band. On 19 December 2005, the SORCE XPS filter mechanism became stuck in position 0 and then several hours later began to operate normally. As a conservative approach for operating its filter mechanism, the SORCE XPS is now primarily making 1-minute solar observations in filter wheel position 6 to maximize its science return (spectral range), and its filter mechanism is operated only occasionally (about monthly) for redundant channel calibration purposes. Only three days of solar observations from either TIMED or SORCE have been lost because of these filter mechanism anomalies.

The accuracy of the XPS solar irradiances is about $20 \%$ as based on pre-flight and inflight calibrations (Woods et al., 1999, 2005, Woods, Rottman, and Vest, 2005). The XPS channels are calibrated about once a year by the TIMED SEE underflight calibration rocket, and the XPS H I Lyman- $\alpha(121.6 \mathrm{~nm})$ irradiances are validated by other SORCE measurements made at $121.6 \mathrm{~nm}$. Prior to the SORCE XPS filter wheel anomaly, redundant channels were used frequently to validate the primary channels. Now, the redundant channels are only used about once a month. Only the XPS Lyman- $\alpha$ channel has degraded on both TIMED and SORCE, at a rate of about $10 \%$ the first year and slower thereafter. Woods, Rottman, and Vest (2005) and Woods and Rottman (2005) provide a detailed description of the SORCE XPS design and calibration methods, irradiance algorithms, and error analysis for the irradiance results. Woods et al. (2005) provide similar information for the TIMED XPS instrument.

\section{Revised XPS Responsivities}

There have been outstanding issues with the XPS responsivities as related to differences between the results of the TIMED XPS calibrations at the German Physikalisch-Technische Bundesanstalt (PTB) electron storage ring BESSY and the results of the SORCE XPS calibrations at the National Institute of Standards and Technology (NIST) electron storage ring 


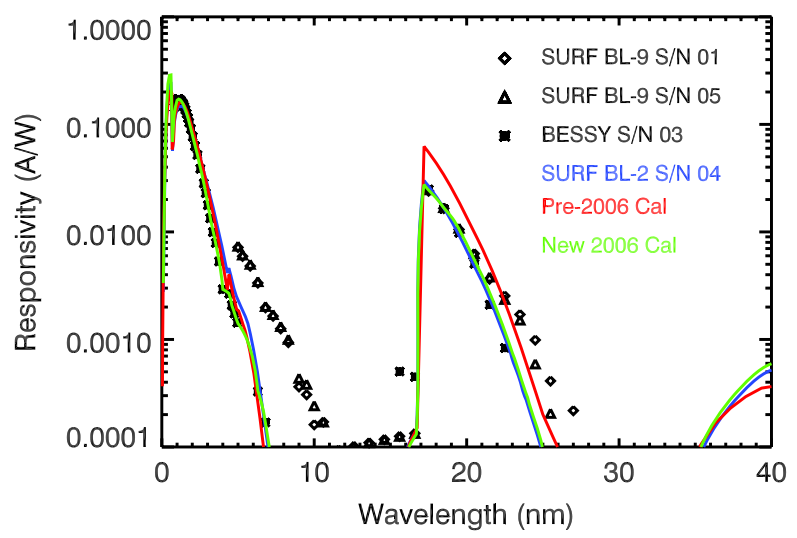

Figure 2 Responsivity calibrations for the $\mathrm{Si}$ photodiodes coated with thin films of $\mathrm{Al}, \mathrm{Nb}$, and $\mathrm{C}$. These calibrations are for different diodes (with serial numbers, $\mathrm{S} / \mathrm{N}$, noted), but these diodes are from the same $\mathrm{Si}$ wafer and are expected to have similar responsivities. The "New $2006 \mathrm{Cal}$ " (green line) is the combination of the various calibrations where they are most accurate. The changes at the short wavelengths, as compared to the "Pre-2006 Cal" (red line), are small. But the new responsivity is much lower than before at wavelengths longer than $17 \mathrm{~nm}$; consequently, the new XPS irradiances are higher than before.

SURF. Woods, Rottman, and Vest (2005) describe in detail these differences, which are a factor of 2 at some wavelengths. The previous XPS data products included the SURF Beam Line 2 (BL-2) calibration results, as they were, at the time, a compromise between the BESSY and SURF Beam Line 9 (BL-9) results.

The revised XPS responsivities now include a composite of the three different calibration results by using the most accurate part from each calibration facility. The PTB BESSY results are generally used in the $1-10 \mathrm{~nm}$ range, and the SURF BL-9 results are generally used in the 17-40 $\mathrm{nm}$ range. Both of these calibrations are based on calibration measurements at a single wavelength using a monochromator with the synchrotron radiation and a reference (calibrated) Si photodiode. Then the SURF BL-2 results are used to fill in the spectral gaps, namely shorter than $1 \mathrm{~nm}$, the $10-17 \mathrm{~nm}$ gap, and longer than the SURF BL-9 results. The SURF BL-2 results are based on the XPS photometers directly viewing the synchrotron radiation (without a monochromator) and then modeling the spectral response by adjusting the filter thickness to match the photometer's signal level. Because SURF BL-2 results rely on a model to provide the spectral response, the SURF BL-2 results are scaled to match the BESSY results at the shorter XUV wavelengths or match the SURF BL-9 results at the longer XUV wavelengths. These different calibration approaches for BESSY and SURF are described in more detail by Woods, Rottman, and Vest (2005).

The actual wavelength boundaries used with BESSY, SURF BL-9, and SURF BL-2 results are selected uniquely for each type of filter, with preference given to the responsivities that are consistent between at least two measurements. As an example, Figure 2 shows the revised calibration and comparisons to BESSY and SURF results for the XPS photometers with the $\mathrm{Al} / \mathrm{Nb} / \mathrm{C}$ filter, one of the primary photometers for the $17-27 \mathrm{~nm}$ range. The BESSY results are used in the $1-7 \mathrm{~nm}$ range as they are consistent with the SURF BL-2 results, and the SURF BL-9 results are used in the 17-20 nm range because they are consistent with both BESSY and SURF BL-2 results. The SURF BL-2 results are used to fill the spectral gaps with a renormalization factor to BESSY and SURF BL-9 results of less than $15 \%$ correction. 
Figure 3 Comparison of new XPS Level 4 to SNOE solar XUV irradiance measurements. The SNOE $17-20 \mathrm{~nm}$ irradiances are a factor of 1.52 higher than those of XPS, and the SNOE 0.1-7 nm irradiances are a factor of 1.61 higher. Although these results are improved from the original SNOE comparisons shown in Woods et al. (2005), there remain differences outside the expected accuracy of about $30 \%$ for each instrument.

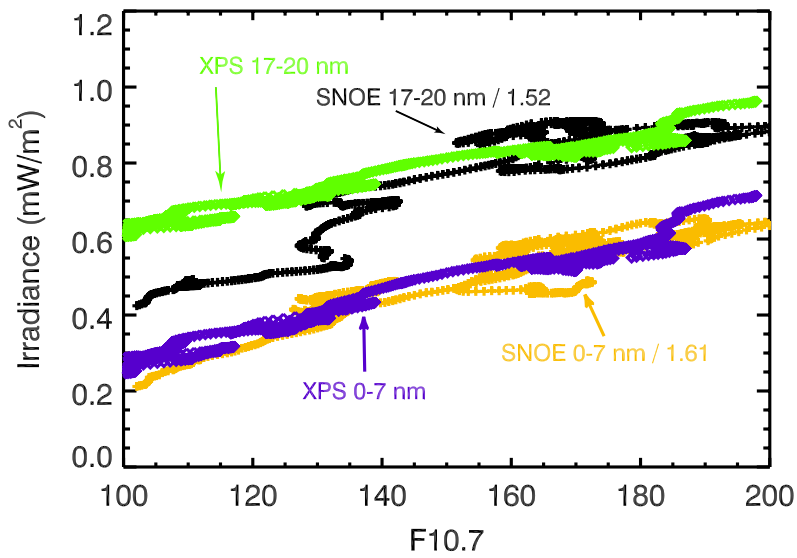

The largest differences in the revised XPS responsivities are at wavelengths longer than $17 \mathrm{~nm}$; consequently, the XPS solar irradiances are most changed in this same wavelength range. The new XPS irradiances agree with the previous version within $10 \%$ for wavelengths shorter than $17 \mathrm{~nm}$, but the new irradiances in the $17-27 \mathrm{~nm}$ range are about 50\% higher. The previous filter model (pre-2006) for the SURF BL-2 measurements was not well optimized and generally had estimated the filter coatings as being too thin. The revised 2006 model for the SURF BL-2 measurements included improvements that produced thicker filter coatings, which are more consistent with the SURF BL-9 results at the XUV wavelengths longer than $17 \mathrm{~nm}$. This improvement in modeling the SURF BL-2 results is related to weighting the results obtained at different synchrotron beam energies. With the previous (pre-2006) model for SURF BL-2 results considered less accurate, the previous XPS solar irradiances in the $17-27 \mathrm{~nm}$ range are considered low.

The new XPS irradiance levels are higher now, but still not as high as the results from SNOE (Bailey et al., 2000, 2001). Woods et al. (2005) show that the SNOE irradiances in the $0-7$ and 17-20 nm bands are a factor of about 1.8 higher than TIMED SEE XPS values using the previous XPS calibration. Figure 3 shows the comparison of SNOE irradiances and the new TIMED SEE XPS results versus the solar 10.7 -cm radio flux proxy (F10.7). This comparison using F10.7 is necessary as the overlap in time with these two missions is short. Our Figure 3 is in the same format as Figure 15 in Woods et al. (2005). The differences between SNOE and XPS are smaller now, with SNOE being a factor of 1.61 higher than XPS for the $0-7 \mathrm{~nm}$ band and a factor of 1.52 higher for the 17-20 nm band. These differences are larger than the expected calibration accuracy of $20-30 \%$ and, as discussed by Woods et al. (2005), probably reflect the accuracy of using different spectral models to analyze the broadband XUV measurements from SNOE and XPS. So which level of the solar XUV irradiance is expected to be more correct? As discussed more in Sect. 5.5, the thermosphere and ionosphere measurements and responses to solar variability are more consistent with the new XPS results.

\section{Improved XPS Level 4 Algorithm}

The original XPS Level 4 algorithm scaled a daily reference spectrum to match the signals (currents) of the XPS photometers and is described in detail by Woods, Rottman, and Vest (2005). The scale factors for the reference spectrum are determined in three bands at $0-4$, 
$4-14$, and $14-27 \mathrm{~nm}$. This approach of having a single reference spectrum for each day to generate a XUV spectrum in 1-nm intervals for each XPS measurement works quite well for nonflare measurements but severely overestimates the flare irradiance by a factor of 2 or more as determined from the measured atmospheric response to flares (e.g., Strickland et al., 2007; Tsurutani et al., 2005). The reason this algorithm breaks down for flares is that the flare spectrum changes significantly, increasing more at the shorter wavelengths. Consequently, this algorithm is expected to overestimate the flare irradiance with a static reference spectrum because the photometers are most responsive at the shorter wavelengths. That is, the photometer signal goes up dramatically, by a factor of about 50 for very large flares, with the real spectral increase expected to be mostly near $2 \mathrm{~nm}$ (Rodgers et al., 2006), but the irradiance calculation uses the lower photometer responsivity at longer wavelengths, which in turn yields high estimates for the flare irradiance.

An improved algorithm makes use of a combination of reference spectra that are representative of both flare and nonflare activity. The CHIANTI version 5.2 spectral model (Dere et al., 1997; Landi et al., 2006) is used to generate reference spectra representative for nonflare and flare measurements. The CHIANTI spectral model includes options with standard differential emission measures (DEMs) and also isothermal spectra appropriate for the Sun. A combination of the standard DEMs is used for the nonflare measurement, considered to be the minimum during each day. Isothermal spectra are used for the flare measurements, which include the XPS signal levels above its daily minimum. The temperature used for the isothermal spectra is derived from the GOES X-Ray Sensor (XRS) measurements of the solar X rays. This technique is similar to that defined by Garcia (1994) in that the ratio of the two XRS measurements provide a monotonic function for temperature, but in the XPS Level 4 algorithm the CHIANTI isothermal models are convolved with XRS responsivities to determine the relationship of the XRS ratio to flare temperature. The new XPS Level 4 algorithm is described by the following equations,

$$
\begin{aligned}
I_{\text {measure }} & =I_{\text {day_min }}+I_{\text {flare }}, \\
I_{\text {day_min }} & =f_{\min } \int_{0}^{\infty} R(\lambda) E_{\min }(\lambda) \mathrm{d} \lambda+f_{\mathrm{AR}} \int_{0}^{\infty} R(\lambda) E_{\mathrm{AR}}(\lambda) \mathrm{d} \lambda, \\
I_{\text {flare }} & =f_{\text {flare }} \int_{0}^{\infty} R(\lambda) E_{\text {flare }}(T, \lambda) \mathrm{d} \lambda, \\
E_{\mathrm{L} 4}(\lambda) & =f_{\min } E_{\min }(\lambda)+f_{\mathrm{AR}} E_{\mathrm{AR}}(\lambda)+f_{\text {flare }} E_{\text {flare }}(T, \lambda) .
\end{aligned}
$$

The measured current ( $I_{\text {measure }}$, in units of $\mathrm{nA}$ ) of the Ti photometers, whose band pass is $0.1-7 \mathrm{~nm}$, is decomposed into the minimum current of the day $\left(I_{\text {day_min }}\right)$ and the flare current $\left(I_{\text {flare }}\right)$, which is the signal above the daily minimum level. It is assumed for this equation that the background (dark) current has already been subtracted, so the $I_{\text {measure }}$ represents just the signal from the solar radiation. The "day_min" current represents the "daily" nonflare component for the spectral model, which is further decomposed in Equation (1b) into a solar cycle minimum contribution $\left(E_{\min }\right.$, in units of $\left.\mathrm{W} \mathrm{m}^{-2} \mathrm{~nm}^{-1}\right)$ and an active region contribution $\left(E_{\mathrm{AR}}\right)$. Each of these contributions, along with the flare contribution $\left(E_{\mathrm{flare}}\right)$, are convolved with the photometer's responsivity $\left(R\right.$, in units of $\left.\mathrm{nA} \mathrm{m}^{2} \mathrm{~W}^{-1}\right)$ and integrated over all wavelengths (in units of $\mathrm{nm}$ ). These two daily components were selected because active regions are the main contribution for coronal variability (e.g., see Woods et al., 2000). The unitless parameters $f_{\min }$ and $f_{\mathrm{AR}}$ are "scale factors" for the solar cycle minimum and active region reference spectra and are determined by matching the XPS signal (current). In concept, $f_{\mathrm{AR}}$ is roughly related to the fractional area of the active regions on the solar 
Figure 4 Example CHIANTI model components for the $0.1-7 \mathrm{~nm}$ irradiance on day 2002/111. The daily components are the quiet Sun (QS) and active region (AR) and are fit to the minimum signal during the day. The flare components are fit to the residual signals above the daily minimum.

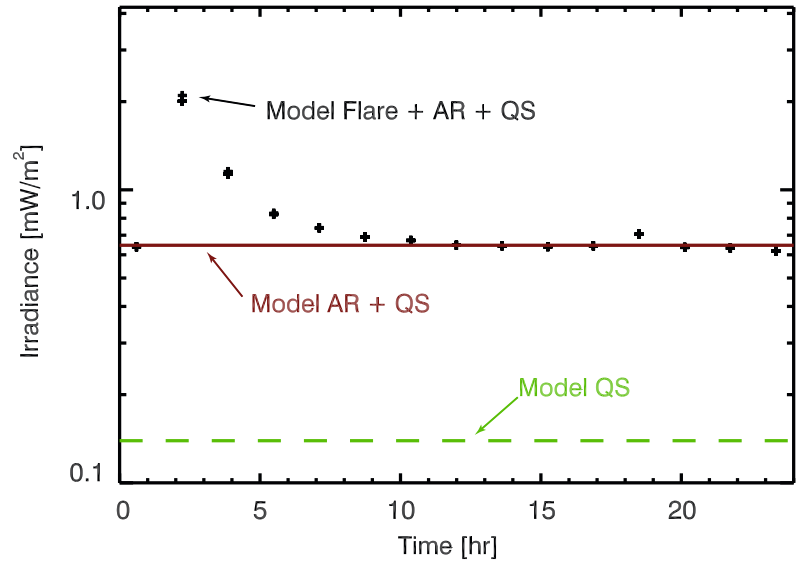

Figure 5 Example GOES X-ray data and derived flare temperature on day 2002/111. The ratio of the GOES $0.1-0.8 \mathrm{~nm}$ irradiance (black line in top panel) to the GOES $0.05-0.4 \mathrm{~nm}$ irradiance (red/gray line in top panel) provides an estimate of the flare temperature (bottom panel) (Garcia, 1994).
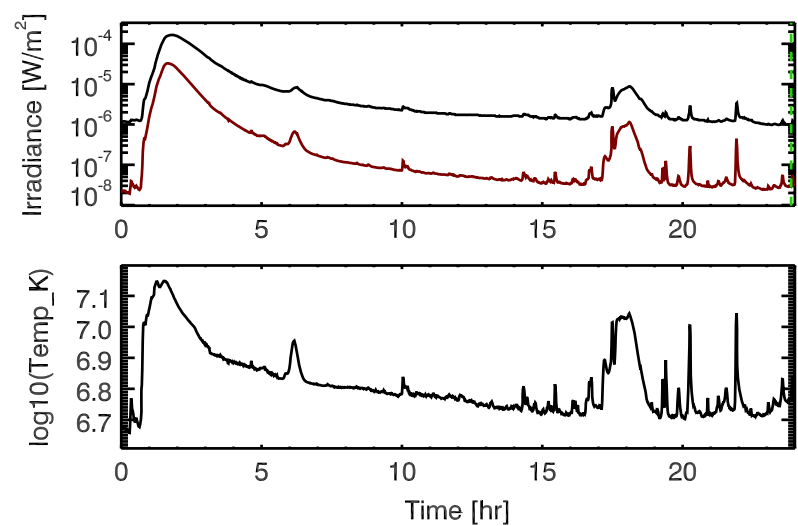

disk. In the algorithm, $f_{\min }$ is set to 1.0 , and $f_{\mathrm{AR}}$ is solved for each day in the XPS Level 4 algorithm. If $f_{\mathrm{AR}}$ ends up negative, then $f_{\mathrm{AR}}$ is set to 0.0 , and $f_{\min }$ is solved, being a solution less than 1.0. The solutions for $f_{\min }$ and $f_{\mathrm{AR}}$ are constant values across a single day, as depicted in Figure 4.

The active region reference spectrum is simply the active region DEM in the CHIANTI spectral model, whereas the solar cycle minimum reference spectrum is a combination of the quiet Sun DEM and coronal hole DEM. Although neither TIMED nor SORCE has observed solar cycle minimum conditions yet, the lowest XPS measurements fit well with the average of the CHIANTI quiet Sun DEM spectrum and coronal hole DEM spectrum, and this average is chosen to be the solar cycle minimum reference spectrum. As solar cycle minimum data are obtained by TIMED and SORCE in the coming year, the solar cycle minimum reference spectrum will be revisited.

The flare reference spectra, $E_{\text {flare }}(T, \lambda)$, are a set of CHIANTI isothermal spectral models where the temperature is determined from the GOES XRS measurements taken with 1-minute cadence. The technique of Garcia (1994), but derived with CHIANTI isothermal spectra, provides the flare temperature relationship from the ratio of the GOES $0.1-0.8 \mathrm{~nm}$ $\mathrm{X}$-ray measurement to the $0.05-0.4 \mathrm{~nm} \mathrm{X}$-ray measurement. As shown for the flare example in Figure 5, this technique indicates that the flare temperature is about $10 \mathrm{MK}$ near the flare peak and then cools down as the flare decays. 
Flares are known to be multitemperature emissions (e.g., Aschwanden, 2007), but the single-temperature approach is a simple, reliable one for data processing because the GOES solar X-ray data are available near real time and because the GOES flare temperature relationship is well characterized (e.g., Garcia, 1994). For processing efficiency, the flare reference spectra, as well as the daily component reference spectra, are static (stored) data files. The temperature $(T)$ grid for these flare reference spectra is from $\log T$ of 4.2 to 8.0 in $0.2 \log T$ steps. The XPS Level 4 algorithm actually uses the two closest temperatures in this grid for the specific temperature calculated from the GOES X-ray data and then determines the flare scale factor, $f_{\text {flare }}$, to match the XPS flare signal, $I_{\text {flare }}$, being simply the signal level above the daily minimum signal level. The use of the flare temperature over $0.2 \log T$ for XPS processing is fortuitously similar to the detailed derivation of the flare DEMs during different flare phases (Chifor et al., 2007). They show that the flare temperature peaks initially near $\log T$ of 7.2, then decays down to a peak of $\log T$ of 6.8 , with the width of the DEMs remaining about $0.2 \log T$. The GOES flare temperature result, with an example shown in Figure 5, has almost the same time variation as the detailed analysis by Chifor et al. (2007). Although this comparison is for just a single flare, the GOES flare temperature result has been examined for many flares, and the flare temperature trend is noted to be similar for most flares.

The primary concerns with the flare component in the XPS Level 4 algorithm are the lack of cool plasma contributions and incomplete coverage for the impulsive phase of the flares. The XPS spectra do have cool plasma contributions from the CHIANTI solar cycle minimum and active region reference spectra (daily components) but none in the flare component. This is because the temperature results from the GOES X-ray ratio yields hot plasma temperatures in excess of $5 \mathrm{MK}$ for almost all conditions. Because the GOES X-ray and XPS $0.1-7 \mathrm{~nm}$ channels primarily have contributions during the gradual phase of the flares, the XPS Level 4 algorithm does not have much, if any, contribution during the impulsive phase of the flares. The Neupert effect (Neupert, 1968) can provide some timing information on the impulsive phase by taking the time derivative of the soft X-ray data from either GOES or XPS, but it does not directly provide quantitative information about the cool plasma contributions during the impulsive phase. Other data, such as cooler emission lines from the TIMED SEE EGS spectra, are needed to help constrain and define the cooler plasma and impulsive phase contributions during flares. However, a critical constraint for the routine data processing of the XPS data is that we are required to release data products within two days of data receipt. That alone eliminates most data sets except for the XPS data itself and the GOES XRS data that are released by NOAA within seconds of data acquisition. Even the EGS results are not useful for routine processing because EGS observations only have a 3\% duty cycle as compared to the SORCE XPS 70\% duty cycle for solar observations. The use of multiple XPS photometers is also useful for a more complete specification of the DEM as done by Rodgers et al. (2006); however, this approach only works for a limited portion of the TIMED and SORCE missions as the XPS filter wheel mechanisms on both missions have had anomalies that required limiting the XPS solar observations to using only one to three photometers. Although more detailed analyses can be performed for a limited number of flare events, some simplifications had to be made (which thus leads to some shortcomings) so that the new XPS Level 4 algorithm could operate over the complete period of both the TIMED and SORCE missions.

The contents of the XPS Level 4 data product includes the modeled spectrum, $E_{\mathrm{L} 4}(\lambda)$, the three scale factors, and the flare temperature results for each measurement of the XPS Ti-coated photodiodes. The cadence of the Level 4 data is the same as the cadence of the XPS measurements, which are typically between 10 and 60 seconds. The reference 
spectra from the CHIANTI model are used at a resolution of $0.002 \mathrm{~nm}$ and in the range from 0.001 to $40 \mathrm{~nm}$, but the XPS Level 4 data product contains the spectra reduced to a resolution of $0.1 \mathrm{~nm}$. The SORCE XPS and TIMED SEE XPS Level 4 data products are stored as NetCDF files (one file per day) and served from the LASP computers at http://lasp.colorado.edu/sorce/ and http://lasp.colorado.edu/see, respectively. In addition, the daily XPS Level 4 data products are merged together over the full mission with different merged data sets for the daily average and for 5-minute averages.

Some of the simplifications made, in part to improve the efficiency of the data-processing algorithms, include (1) having no center-to-limb variation for the CHIANTI model radiances, (2) using the measured current from the most reliable (single bandpass) Ti-coated photodiodes in XPS, and (3) using a single temperature for the flare spectrum, as already mentioned. There were initial concerns that a single photodiode, in this case the Ti-coated photodiodes with a bandpass of $0.1-7 \mathrm{~nm}$, would not be able to predict spectral irradiance at other wavelengths. Algorithm tests with the other XPS photodiodes showed that the daily component results, namely the scaling factors for solar cycle minimum and active regions, are similar for all of the XPS photodiodes. This result suggests that the CHIANTI spectral models are a good representation across the XPS spectral range of 0.1 to $27 \mathrm{~nm}$. Furthermore, as shown in the next section, the XPS Level 4 spectral results at wavelengths longer than $27 \mathrm{~nm}$ agree very well with the spectrally resolved measurements by TIMED SEE EGS in the $27-40 \mathrm{~nm}$ range. Considering that the CHIANTI isothermal models for the hot corona temperatures have the largest variations at the shorter wavelengths, the XPS Ticoated photodiode ( $0.1-7 \mathrm{~nm}$ bandpass) is the best diode suited for flare detection and most appropriate for use in the XPS Level 4 algorithm. More details are given in the next section, where some results for the new XPS Level 4 data products are described and comparisons to other measurements and models are provided.

\section{Results}

The new XPS Level 4 data products that incorporate the combination of daily components and a flare component of the CHIANTI spectral models are improvements over the previous version. The flare reference spectra are uniquely different than the daily (nonflare) reference spectra, and the temperature for the flare spectra are based on the well-characterized GOES solar X-ray measurements, whereas the previous XPS version used a single reference spectrum for each day, which did change from day to day to account for solar activity but did not have a flare component. Because of the more realistic flare reference spectra, the new XPS flare results, as shown later, are consistent for the first time with the atmospheric response to flares (e.g., Strickland et al., 2007). This discrepancy was the original motivation for improving the XPS Level 4 algorithm. While evaluating the results in the following subsections, one does need to keep in mind that the XPS Level 4 data products are actually a combination of high-resolution spectra from the CHIANTI model and that the XPS direct measurements are broad band with only $7-10 \mathrm{~nm}$ resolution. In other words, the irradiance over broad ranges $(\approx 10 \mathrm{~nm})$ is considered much more accurate than the accuracy of the irradiance at higher resolution.

\subsection{Solar Variability}

The solar irradiance variations can generally be decomposed into the short-term flare variations lasting minutes to hours, the 27-day solar rotation variations, and the 11-year solar 
cycle variations. The solar XUV radiation is highly variable on all of these time scales and is well known for flare monitoring at the shorter wavelengths using the NOAA GOES solar $\mathrm{X}$-ray measurements. The XUV range is dominated by coronal emissions that include atomic (bound - bound) emission lines and bremsstrahlung (free - free) continuum, and these coronal emissions are enhanced through the dynamic magnetic activity on the Sun. Magnetic reconnection in the corona is thought to be the energy source for flare events (e.g., Aschwanden, 2004), and the magnetic-driven active regions, and their subsequent decay into active network, are the source for most of the longer term (days to years) variations (e.g., Woods et al., 2000). The XPS measurements clearly show these types of variations with a factor of 2 to even more than a factor of 100 at the shortest wavelengths seen during flare events and over the longer term.

TIMED SEE XPS and SORCE XPS have produced stunning new observations of irradiance increases in solar flares, at longer wavelengths than the NOAA GOES standard flare monitoring in the 0.05 - to $0.8-\mathrm{nm}$ wavelength range. Intense solar storms - including some of the largest in the space era - occurred during the TIMED and SORCE missions, for example in April 2002, July-August 2002, May - June 2003, October-November 2003, November 2004, January 2005, July 2005, September 2005, and December 2006. The October-November 2003 storms produced record solar flares with an X28 flare on 4 November 2003 and an X17 flare on 28 October 2003.

One of the most important results from the XPS measurements is the finding that the XUV variations during large flares are larger than their expected solar cycle variations. Examples of the flare and solar cycle variations are shown in Figure 6 in 0.1-nm intervals as given by the XPS Level 4 data products. The flare example is for the X28 flare on 4 November 2003, the largest flare seen during the TIMED and SORCE missions. The solar cycle example uses the results on day 2002/235 (F10.7=230) and day 2006/265 (F10.7=73) for solar cycle maximum and minimum, respectively. The solar cycle 23 variation is expected to be somewhat higher as the next solar cycle minimum may not occur until sometime in 2008. Nonetheless, the flare variation is significantly more than the solar cycle variation at wavelengths shorter than $16 \mathrm{~nm}$ by more than a factor of 10 at some wavelengths. At longer wavelengths, the flare variation is more similar to the solar cycle variation but with some emission lines showing more variability for the flare event. As discussed further in Sect. 5.3, the XPS Level 4 solar cycle variation in the $27-40 \mathrm{~nm}$ range is consistent with the TIMED SEE EGS spectral measurements.

The XPS flare results also provide information about the total energy from a flare. The simultaneous observations with the SORCE and TIMED SEE instruments have provided new insight into the spectral irradiance variability during flares over the full ultraviolet range from 0.1 to $200 \mathrm{~nm}$, including the first definitive flare detection in the total solar irradiance (TSI) record by the SORCE Total Irradiance Monitor (TIM) (Woods et al., 2004b). Additionally, the TIM and XPS measurements of several flares indicate that the total energy from the flares is about 10 times larger than previous estimates (Woods, Kopp, and Chamberlin, 2006). Furthermore, Woods, Kopp, and Chamberlin (2006) showed that the relationship of the total energy of a flare to the energy in the XUV range depends on the location of the flare. Namely, the total flare energy seen at Earth was essentially the same as the energy in the XUV range if the flare is near the solar limb, but the total flare energy was about four times more than the energy in the XUV range for flares near solar disk center. This dependence on flare location is obviously related to the radiative transfer through the solar atmosphere whereby the XUV wavelengths are optically thin and the longer wavelengths are more optically thick and thus absorb more for flares near the limb. Woods, Kopp, and Chamberlin (2006) provide this flare energy relationship using a previous version of XPS 

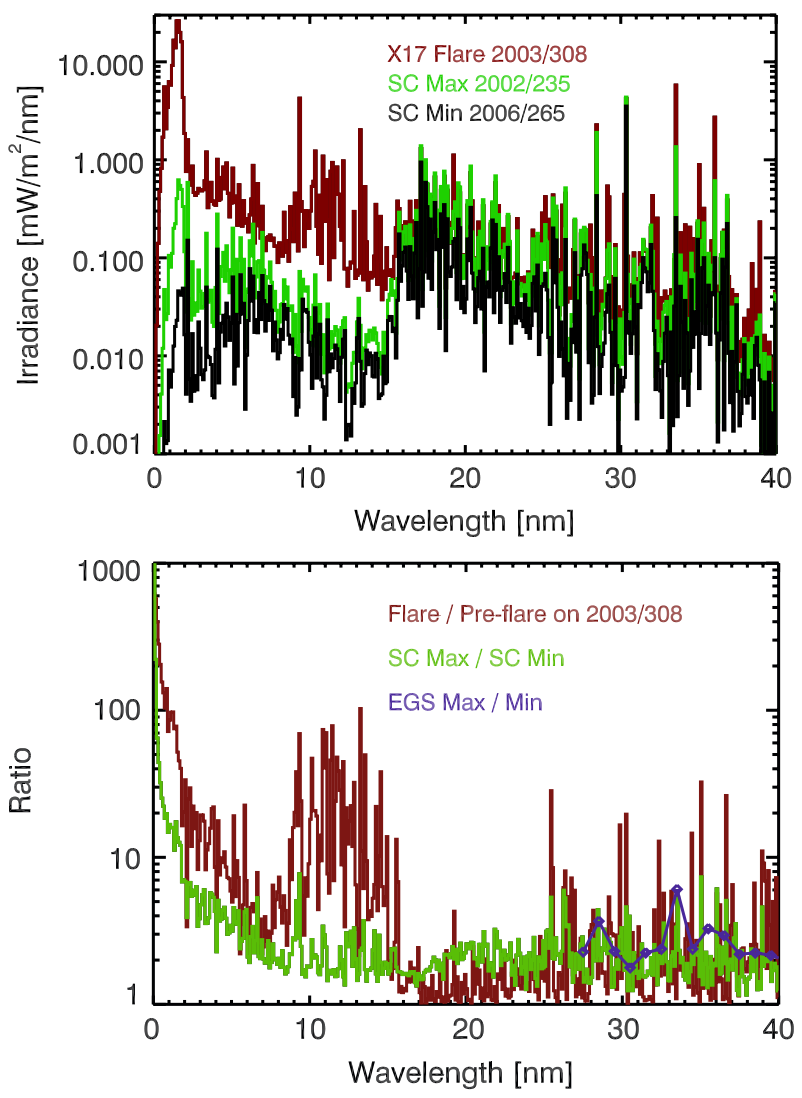

Figure 6 Example XPS Level 4 results at 0.1-nm resolution for solar cycle (SC) variations and for the X28 flare on 4 November 2003. The top panel shows the irradiance for three different times, and the bottom panel shows the irradiance ratios. The solar cycle and flare variations longer than $15 \mathrm{~nm}$ are similar in spectral shape and are about a factor of 2. This flare variation is even more than the solar cycle variation at shorter than $15 \mathrm{~nm}$. The SEE EGS solar cycle results at longer than $27 \mathrm{~nm}$ are in good agreement with XPS results.

data, and the results with the new XPS Level 4 product in the $0.1-27 \mathrm{~nm}$ range is, on average, a factor of 0.23 of the previous version. Although the Woods, Kopp, and Chamberlin (2006) results are still valid for the total flare energy and the relationship of the total flare energy to the GOES $0.1-0.8 \mathrm{~nm}$ X-ray flare energy, the XUV results in this paper need to be reduced by this factor of 0.23 . Consequently, the XUV $(0.1-27 \mathrm{~nm})$ contributions to the total flare energy are now reduced to about $7 \%$ for flares near disk center and to about $20 \%$ for flares near the limb. The Woods, Kopp, and Chamberlin (2006) conclusion that there is significant flare energy coming from wavelengths longer than $200 \mathrm{~nm}$ is still true, but even more so for all flares, even flares near the limb.

\subsection{XPS Level 4 Scale Factors}

The scale factors in the XPS Level 4 algorithm in Equation (1) are the factors used to scale the reference spectra to match the XPS measurement, and they indicate solar variability as shown in Figure 7 . The daily $f_{\min }$ is expected to be 1.0 , but it does vary less than 1.0 when 


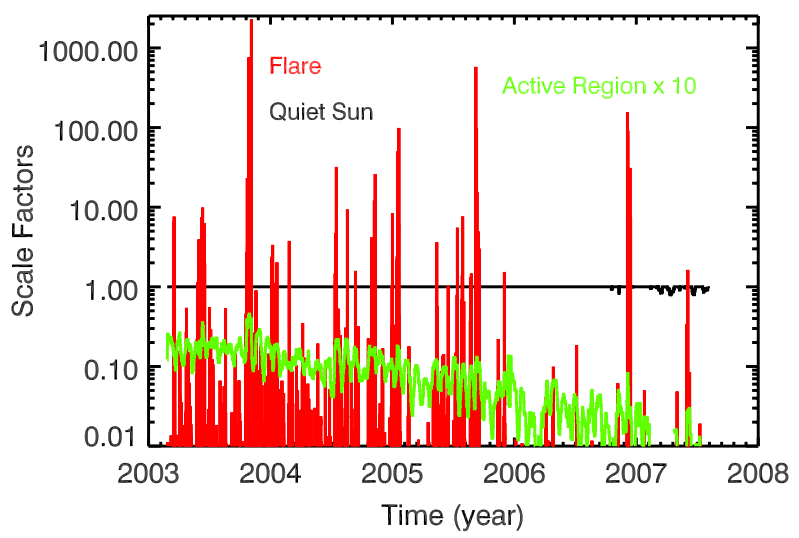

Figure 7 Model scale factors in the SORCE XPS Level 4 data products. The quiet Sun scale factor (flat black line) should always be 1.0 except when the XPS irradiance drops below the solar cycle minimum reference model level. The active region scale factor (green/gray line) represents, in concept, the fraction of the disk covered by active regions and more clearly shows the variations associated with 27-day solar rotation modulation of the active regions facing toward Earth and the long-term changes associated with the 11-year solar cycle. The flare scale factor (red/dark gray line) indicates the magnitude of the flare irradiance and is an indication of modest and large flares when above a factor of about 2.

near solar minimum conditions. This result indicates that the reference minimum spectrum is slightly too large. Of course, the calibration for the XPS photometer also influences the $f_{\min }$ scale factor, but the selection of the reference minimum spectrum, in concept, should be tuned to give a value of 1.0 even at solar cycle minimum. This minimum spectrum was taken as the average of the CHIANTI quiet Sun and coronal hole reference spectra, so adding more of the coronal hole reference spectrum would improve the reference minimum spectrum for the XPS Level 4 algorithm.

The daily $f_{\mathrm{AR}}$ scale factor is roughly related to the fractional area on the solar disk that is covered by active regions. The XPS active region scale factors shown in Figure 7 are $2-4 \%$ during solar cycle maximum. This result is a factor of about 2 less than the combined plage and decayed plage fractional area derived from Ca II K and He II $30.4 \mathrm{~nm}$ solar images (Worden, White, and Woods, 1998; Worden et al., 1999). This difference is not as large as it might first seem. The CHIANTI active region spectrum is more appropriate for plage center, but the Worden, White, and Woods (1998), Worden et al. (1999) results correspond to the full plage area that effectively reduces the mean plage intensity. In other words, Worden, White, and Woods (1998), Worden et al. (1999) has lower plage intensity and larger plage areas; whereas the XPS/CHIANTI algorithm, with its higher plage intensity, is expected to represent smaller plage areas. Quantifying these differences more precisely is not feasible as the XPS results are not based on solar images. Nonetheless, the XPS Level 4 scale factor for the active region behaves as expected in that it clearly shows 27-day solar rotation variations and is near zero for low solar cycle activity.

The flare scale factor varies the most, with its value ranging near zero most of the time to over a factor of 10 for the larger flares. The largest flare factor is 2300 for the X28 flare on 4 November 2003. The flare activity is often clustered as associated with one or two active regions as they rotate across the solar disk. There have been two to four major clusters (solar storm periods) each year during the SORCE mission starting in 2003 near solar maximum, but with fewer storms in 2006-2007 when near solar minimum. 


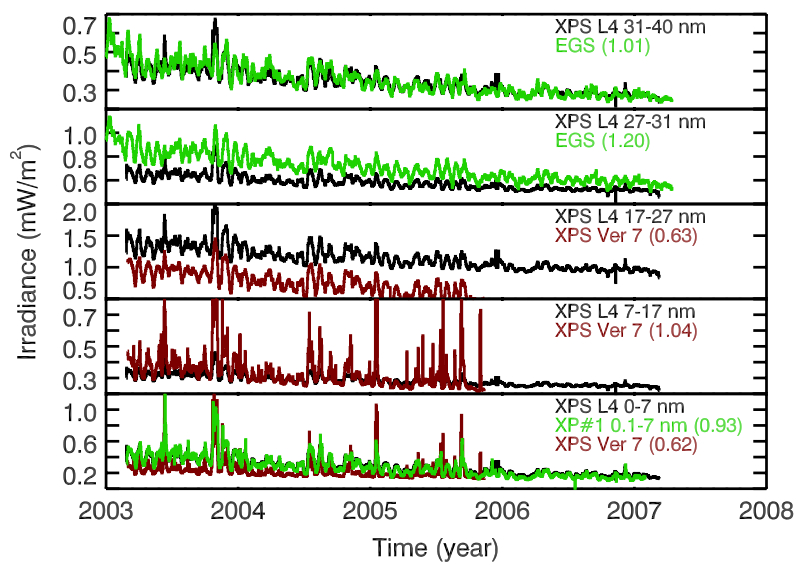

Figure 8 Comparisons of new SORCE XPS Level 4 daily averaged results (black lines) in broad bands to earlier version 7 of SORCE XPS data (red lines) and to SEE EGS measurements (green lines in top two panels). The number in parentheses is the ratio of that item to the new XPS result. The CHIANTI model is not adequate for the optically thick He II $30.4 \mathrm{~nm}$ emission, as reflected in the $27-31 \mathrm{~nm}$ comparison to EGS. The offsets between the new XPS and version 7 data is due to revised responsivities in the new version. One will also notice that the previous version 7 results in the $7-17 \mathrm{~nm}$ band had unrealistically large variations from day to day.

\subsection{Comparisons to Other Measurements}

A primary validation for the new XPS Level 4 algorithm (using CHIANTI reference spectra) is the comparison to the TIMED SEE EGS measurements made at wavelengths longer than $27 \mathrm{~nm}$ and with 0.4-nm spectral resolution. The spectral overlap between XPS Level 4 and EGS is 27 to $40 \mathrm{~nm}$. Figures 8 and 9 show this comparison for the full SORCE mission and for the large flare on day 2003/308. These plots compare to EGS in the spectral bands of $27-$ 31 and $31-40 \mathrm{~nm}$. The agreement in the $31-40 \mathrm{~nm}$ band is excellent, with the XPS Level 4 product being only $1 \%$ higher, on average, than EGS measurements. The more detailed spectral comparison in the 31-40 nm range, as given in Figure 1, shows larger differences than the integrated band differences. Nonetheless, this comparison shows surprisingly good agreement when one considers that the XPS Level 4 result is the CHIANTI reference spectra that are scaled to agree with the XPS broadband measurement in the $0.1-7 \mathrm{~nm}$ band. This excellent agreement in the $31-40 \mathrm{~nm}$ range gives some confidence that the CHIANTI reference spectra (and thus XPS Level 4 products) in the 7-27 nm range are realistic solar spectra.

The XPS Level 4 product in the $27-31 \mathrm{~nm}$ band is $21 \%$ lower than the EGS measurements, and this difference is due primarily to the CHIANTI model not properly treating the optically thick He II $30.4 \mathrm{~nm}$ emission. That is, the CHIANTI model has He II $30.4 \mathrm{~nm}$ intensity values in the reference spectra that are too low. This is clearly evident by a detailed comparison with an EGS spectrum (with EGS Level 2 being in 0.1-nm intervals) and is also obvious in the comparison time series that show less solar rotational variation in the XPS Level 4 product than the daily EGS measurements. There is also much less flare variation of the He II $30.4 \mathrm{~nm}$ emission in the XPS Level 4 product, but this reduced flare variation can be the result of the XPS flare component not having a cool plasma contribution. The variability of the nearby $\mathrm{Si} \mathrm{XI}$ emission at $30.3 \mathrm{~nm}$ might also contribute to this difference, but this emission is not expected to be the primary cause because it is weaker than the He II 
Figure 9 Comparisons of new SORCE XPS Level 4 results (diamond symbols) on day 2003/308 in broad bands to earlier version 7 of SORCE XPS data (triangle symbols) and to SEE EGS measurements (square symbols). As noted for Figure 8, the optically thick He II $30.4 \mathrm{~nm}$ emission is not well modeled for the XPS Level 4 product as reflected in the $27-31 \mathrm{~nm}$ comparison to EGS. The previous version 7 XPS results showed more variability during flares; the new version is considered the more correct variation for flares.

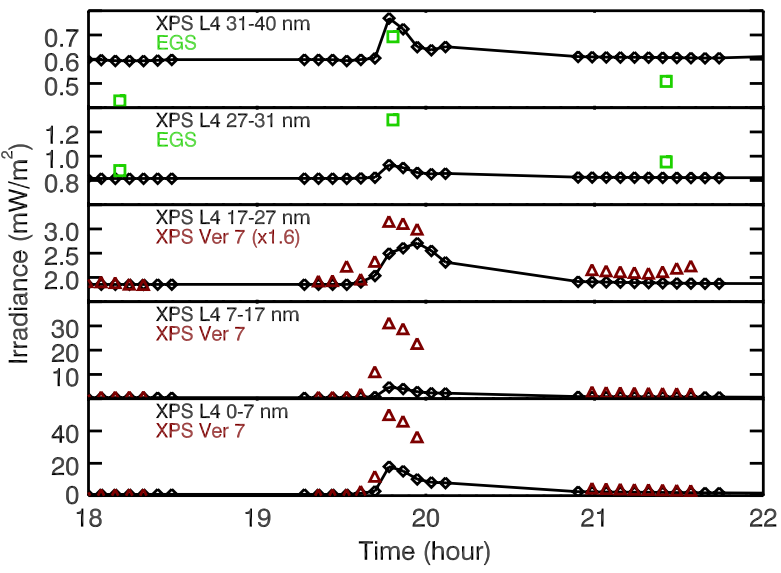

emission and because the other CHIANTI model coronal emissions in the $27-40 \mathrm{~nm}$ range have reasonable agreement with the EGS spectral measurements. A future version of XPS Level 4 algorithm could fix this shortcoming of the CHIANTI model by manually inserting the EGS results into the reference spectra for the He II $30.4 \mathrm{~nm}$ emission line.

The other comparisons shown in Figures 8 and 9 are comparisons of the new SORCE XPS Level 4 product (version 8) to the older XPS spectral product (version 7). These comparisons are not intended to be shown as a validation but instead are shown to highlight the differences in results between the older XPS spectral algorithm and new algorithm that uses the CHIANTI spectral models. The main motivation for switching from the old algorithm (that scaled a daily solar spectrum to match the XPS data) was to get more realistic flare spectra from the XPS measurements. With the new XPS Level 4 product, the flare variations are, as expected, much less at the shorter wavelengths, as shown in Figure 9. In comparing more than 500 flare measurements, the ratios of the XPS old version 7 flare data to new version 8 data are 2.1 and 3.5 for the $0.1-7$ and $7-17 \mathrm{~nm}$ bands, respectively. In contrast with this change, the XPS old version 7 flare data in the $17-27 \mathrm{~nm}$ band is less by an average factor of 0.77 than the new version 8 data. In other words, the new XPS results have significantly less flare variability in the $0.1-17 \mathrm{~nm}$ range but slightly more in the $17-27 \mathrm{~nm}$ range than the old version.

There are also systematic differences between the SORCE XPS version 7 and 8 data sets. The ratios of the XPS version 8 daily averaged data to version 7 data are $0.62,1.04$, and 0.63 for the $0.1-7,7-17$, and 17-27 nm bands, respectively. Most of these differences can be explained by the updated calibration used in XPS version 8 processing (see Sect. 3), but some of these differences are possibly related to the spectral shape differences in the model reference spectra.

The flare comparison in Figure 9 shows similar variations as measured by EGS at wavelengths longer than $27 \mathrm{~nm}$. The flare component in the XPS Level 4 product is created by using a hot corona temperature, so, as shown in Figure 6, the flare contribution dominates the irradiance results for wavelengths shorter than $17 \mathrm{~nm}$ but still provides a modest contribution at longer than $17 \mathrm{~nm}$ from the hot corona bremsstrahlung continuum and from a few hot corona emission features.

The SOHO Solar EUV Monitor (SEM) (Judge et al., 1998) measurements also overlap with the XPS Level 4 spectral range. The SEM first-order measurements in the $26-34 \mathrm{~nm}$ band have been compared to SEE EGS measurements and show about $20 \%$ difference, with 
Figure 10 Comparison of TIMED SEE XPS Level 4 results to the Rodgers et al. (2006) model results for the large X28 flare on 4 November 2003. Both results are based on CHIANTI spectral models but using very different techniques: Rodgers et al. (2006) performed a more complete analysis of the flare DEM and the XPS Level 4 algorithm uses an isothermal CHIANTI model for the flare. The similarity of the spectral shapes of the two results validates the simple technique used in the XPS Level 4 algorithm.

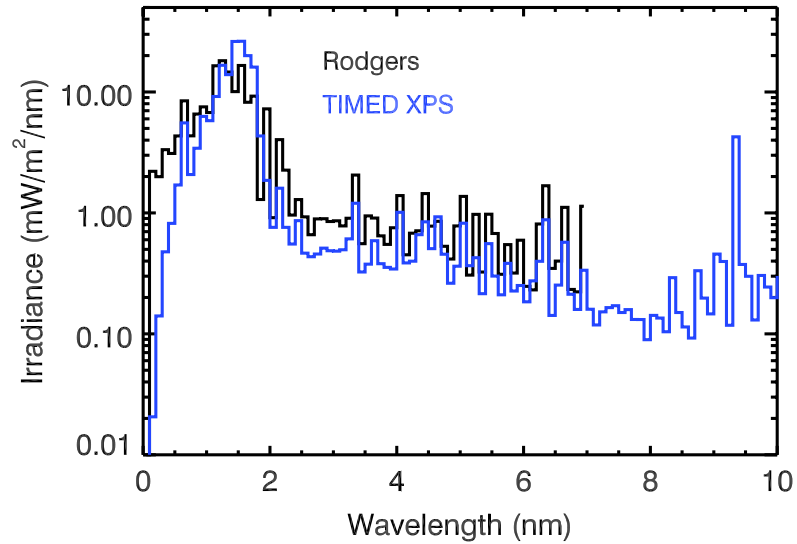

SEM being higher than SEE (Woods et al., 2005). The SEM zeroth-order measurement in the $0.1-50 \mathrm{~nm}$ band is even broader than that of XPS, and the processing of SEM data into irradiances currently only uses a single solar spectrum during the full SOHO mission. Nonetheless, the SEM $0.1-50 \mathrm{~nm}$ irradiance result agrees reasonably well with the SEE and XPS results for a daily comparison (Woods et al., 2005; Woods, Rottman, and Vest, 2005). The comparison of new XPS Level 4 results are about the same as the earlier comparisons because the SEM $0.1-50 \mathrm{~nm}$ band is so broad and the XPS changes in responsivity are mostly limited to $17-27 \mathrm{~nm}$.

\subsection{Comparison to Another Spectral Model}

These XPS Level 4 results can be compared to another spectral model that has been applied to the XPS data. Rodgers et al. (2006) describe one technique and results from deriving the DEM for $\log T$ between 6.5 and 8.0 using multiple XPS channels and then calculating the CHIANTI spectral model with this DEM. An example of this comparison is shown in Figure 10 for day 2003/308 for the large X28 flare near 19:47 UT.

Comparisons between Rodgers (2006; hereafter RODGERS) results and XPS Level 4 near the peaks of flares indicate similar spectral shapes for the irradiance and also similar integrated $0-7 \mathrm{~nm}$ irradiances. Because both use the CHIANTI spectral model, the spectral shape differences are surely related to the two different techniques. The XPS Level 4 algorithm has a known weakness of just using a single temperature for the flare spectrum, and the RODGERS technique is weak in not specifying the DEM for $\log T$ below 6.5. So it is not clear which technique is better at describing the real solar spectrum during flares. This issue is not expected to be fully addressed until there are spectral measurements from the Solar Dynamics Observatory (SDO) Extreme ultraviolet Variability Experiment (EVE), with its launch planned in late 2008 (Woods, Lean, and Eparvier, 2006). The differences for the integrated $0-7 \mathrm{~nm}$ irradiances are about $35 \%$, but this is limited to the comparison of just three flare events. Nonetheless, this difference is about the same magnitude of the accuracy of the XPS measurements, so it is reasonable to assume that RODGERS and XPS Level 4 irradiance results have about $40 \%$ accuracy during flares, and somewhat better for nonflare results.

Both of these results use the same CHIANTI spectral model, albeit different DEM functions. Because the latest version of the CHIANTI model has more emission lines in the XUV range, the CHIANTI spectral model is considered to be slightly better than 
the other spectral models, such as the Mewe model (Mewe and Gronenschild, 1981; Mewe, Gronenschild, and van den Oord, 1985; Mewe, Lemen, and van den Oord, 1986), Mewe - Kaastra - Liedahl (MEKAL) (Liedahl, Osterheld, and Goldstein, 1995) or the Astrophysical Plasma Emission Code (APEC) model (Smith et al., 2001). Again, the choice of spectral model and knowledge of the true solar spectral distribution over the full XUV range can be more directly accessed once there are SDO EVE measurements with its 0.1-nm spectral resolution.

\subsection{Comparison to the HEUVAC Model}

Comparison of the XPS results to a solar irradiance empirical (proxy) model also has value in that the empirical model represents previous measurements. Such comparisons for the XPS data have been given by Woods et al. (2005), Woods, Rottman, and Vest (2005) for the NRLEUV model (Warren, Mariska, and Lean, 2001), SOLAR2000 model (Tobiska et al., 2000), EUV81 model (Hinteregger, Fukui, and Gilson, 1981), and EUVAC model (Richards, Fennelly, and Torr, 1994). Briefly here, the EUV81 model had the largest differences, the SOLAR2000 model agreed well at most wavelengths partly because it was derived with the TIMED SEE measurements, the NRLEUV model agreed well in the $25-100 \mathrm{~nm}$ but was low at wavelengths shorter than $25 \mathrm{~nm}$, and the EUVAC model agreed reasonably well at all wavelengths.

The model comparison here is with the EUVAC model (Richards, Fennelly, and Torr, 1994) because it is an empirical model based entirely on previous measurements and because it compared well with the previous XPS measurements and also with atmospheric modeling (Solomon and Qian, 2005). More recently, the EUVAC model has been updated to be at higher spectral resolution and renamed the HEUVAC model (Richards, Woods, and Peterson, 2006). The EUVAC and HEUVAC models use F10.7 as the proxy and thus can only estimate the solar irradiance for daily averages. As verification of both HEUVAC and XPS Level 4 results, these two are compared for spectral differences and also for variations over the solar cycle. The scaled F74113 reference spectrum is used for the HEUVAC model in these comparisons, and this scaling is a factor of 2 or more for wavelengths shorter than $25 \mathrm{~nm}$.

For spectral comparisons, the XPS results for the solar cycle variations given in Figure 6 are compared to the HEUVAC model estimates. These dates are 2006/265 for solar cycle minimum and 2002/235 for maximum and are only chosen to be representative of low and high activity, not to be considered the true extremes for solar cycle 23. Figure 5.5 shows the comparisons for these two dates. The 0.1-nm resolution spectra in Figure 5.5A indicate that the HEUVAC model has many bins with very low levels (red dots), but otherwise the irradiance levels are similar. These low bins in HEUVAC are unrealistic as the CHIANTI spectra have emissions at all bins. The F74113 spectrum used by HEUVAC has 969 emissions at wavelengths shorter than $105.5 \mathrm{~nm}$ and has 304 zero (low) values when combined into 0.1-nm bins. Therefore, the ratios of HEUVAC to XPS Level 4 results are shown at $1-\mathrm{nm}$ resolution. The ratios for the integrated irradiance from 0.1 to $40 \mathrm{~nm}$ are very good, being 0.89 for cycle minimum and 1.29 for maximum. However, there are large systematic differences between these spectra, with some of the largest differences being shortward of $2,8.5,14.5,24.5 \mathrm{~nm}$ and longer than $37 \mathrm{~nm}$. The $0.1-40 \mathrm{~nm}$ solar cycle variation is larger for the HEUVAC model than the XPS L4 result, but the XPS L4 results have larger solar cycle variation at wavelengths shorter than $7 \mathrm{~nm}$.

The other comparison is the temporal variations over the solar cycle as illustrated in Figure 12. The four bands chosen for this comparison are $0-5,5-17,17-27$, and $27-40 \mathrm{~nm}$. 

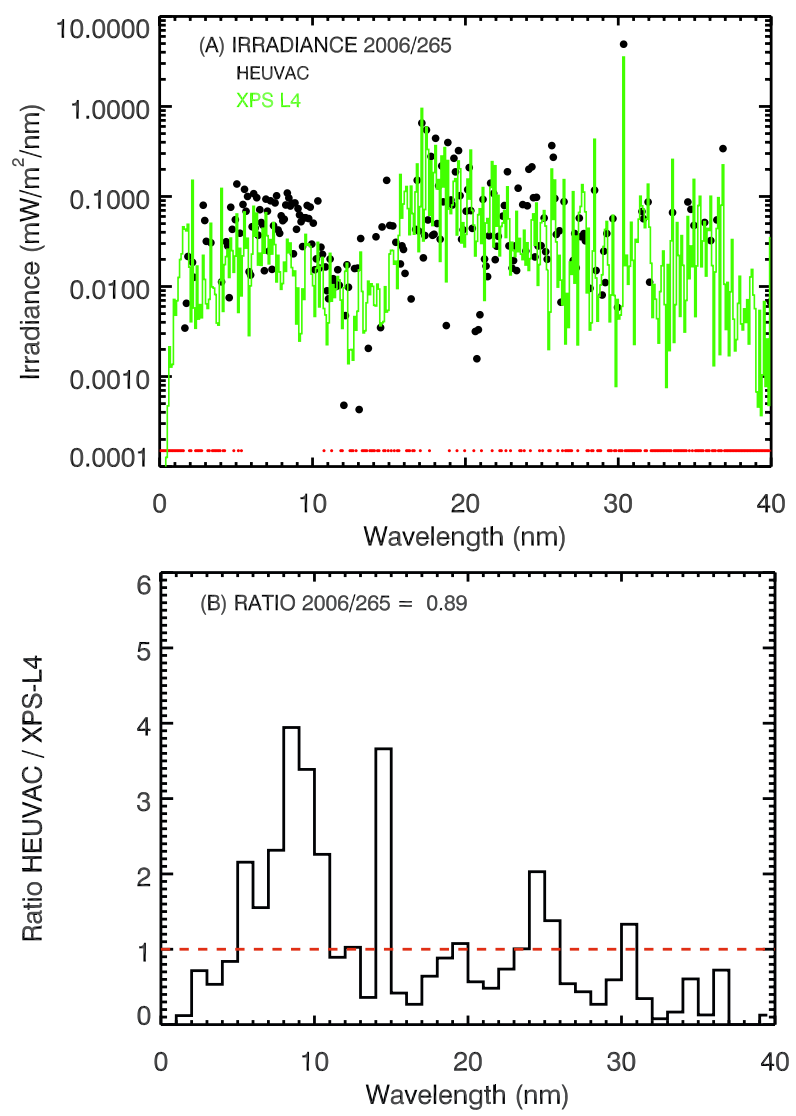

Figure 11 Comparison of HEUVAC model estimates to XPS Level 4 spectra for solar cycle maximum day $2002 / 235$ and minimum day 2006/265. The spectra in panel A are shown at 0.1-nm resolution, and the ratios are shown at 1-nm resolution. The small dots in panel A are the 0.1-nm bins in the HEUVAC model with low values. Although the average over $0.1-40 \mathrm{~nm}$ is less than $30 \%$, there are large systematic differences between these spectra as shown in panels B and D. The $0.1-40 \mathrm{~nm}$ solar cycle variation (panel C) is larger for the HEUVAC model than the XPS L4 result, but the XPS L4 results have larger solar cycle variation at wavelengths shorter than $7 \mathrm{~nm}$.

The bands with the most difference are the $0-5$ and $5-17 \mathrm{~nm}$ bands. The HEUVAC $0-5 \mathrm{~nm}$ band has lower intensity and much less solar cycle variability than the XPS Level 4 results, but we note that the HEUVAC and XPS agree better near solar cycle minimum. Because the XPS Level 4 algorithm is based primarily on the XPS 0.1-7 nm photometer, it suggests that the HEUVAC model at wavelengths shorter than $5 \mathrm{~nm}$ needs to be updated, primarily for its sensitivity to solar variations. The other large difference is that the HEUVAC model is higher than the XPS Level 4 result in the $5-17 \mathrm{~nm}$ band by a factor of 1.38 . As will be shown in the next section, the Fast Auroral Snapshot (FAST) photoelectron measurements suggest that the XPS Level 4 results are low near $10 \mathrm{~nm}$; therefore, the XPS Level 4 algorithm and potentially the CHIANTI spectral models probably need to be updated within this 5-17 nm band. The HEUVAC and XPS ratio in this band does not have much of a slope over the solar cycle; thus the $5-17 \mathrm{~nm}$ differences are mostly a systematic offset. The 

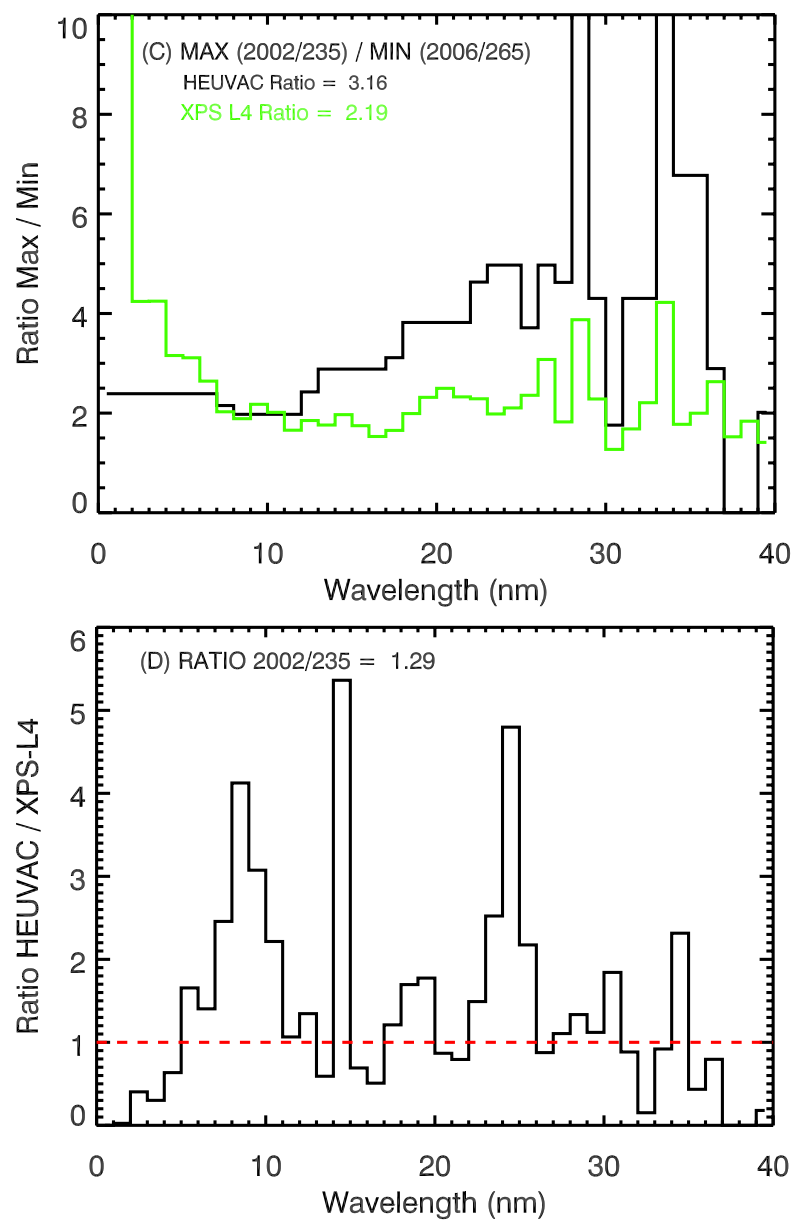

Figure 11 (Continued)

other two bands at 17-27 and 27-40 nm show differences over the solar cycle, but their systematic offsets are small as their mean ratios are near 1.0. For both of these bands, the HEUVAC model estimates more solar cycle variations than the XPS Level 4 result. Because the EGS spectral measurements show solar cycle variation very similar to the XPS results at wavelengths longer than $27 \mathrm{~nm}$, the HEUVAC model could be updated to have less solar cycle variation. In summary, the HEUVAC model may need to be updated at wavelengths shorter than $5 \mathrm{~nm}$ to increase its irradiance level and to have larger solar variations and revised between 17 and $40 \mathrm{~nm}$ to have less solar variations. For XPS, the region between 5 and $17 \mathrm{~nm}$ may need to be updated with higher irradiance levels.

\subsection{Comparisons to Atmospheric Responses}

The upper atmosphere responds to solar variation, and these responses include changes to the temperature, neutral density, plasma (electron) density, and composition. These solardriven changes are expected to be consistent with the solar variations at the appropriate 
Figure 12 Comparison of HEUVAC model estimates to XPS Level 4 time series for $0-5$, $5-17,17-27$, and $17-40 \mathrm{~nm}$ bands. The top panel shows the irradiances for the $0-5$ and $5-17 \mathrm{~nm}$ bands as they differ the most. The bottom panels shows the ratio of HEUVAC bands to the XPS Level 4 bands.

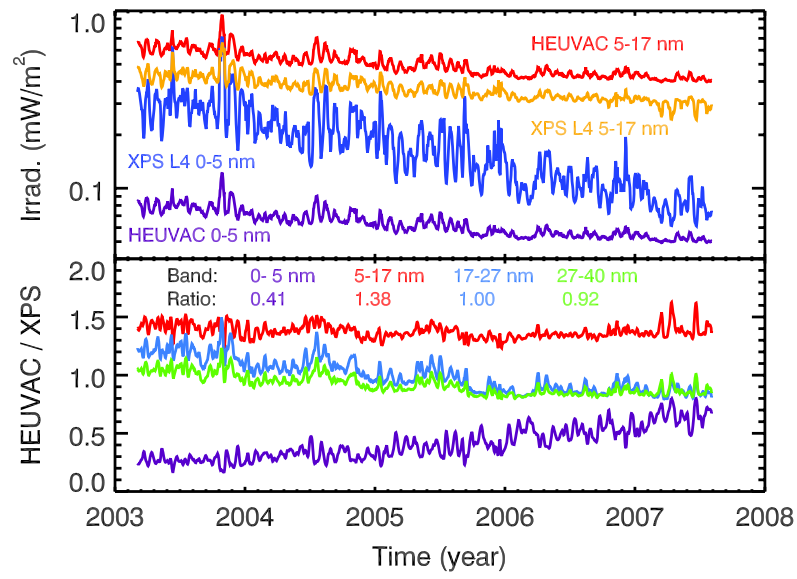

wavelengths. The processes driving these changes are all wavelength dependent in both the cross sections in the atmosphere and the solar spectrum. The changes in the temperature, neutral density, and composition are primarily driven by the longer wavelengths in the EUV and far-UV (FUV) ranges, which are not measured by XPS.

The creation of the ionosphere, especially at lower latitudes, is by the solar spectrum shorter than about $100 \mathrm{~nm}$ through photoionization of $\mathrm{O}, \mathrm{N}_{2}$, and $\mathrm{O}_{2}$ in the thermosphere from about $100 \mathrm{~km}$ up to about $400 \mathrm{~km}$. The fraction of the solar radiation going into the different species is shown in Figure 13 for both solar cycle minimum and maximum conditions. This result represents the global average absorption from using the neutral densities from the NRLMSISE-00 atmospheric model (Picone et al., 2003) and photoionization and photodissociation cross sections of $\mathrm{H}, \mathrm{O}, \mathrm{N}_{2}$, and $\mathrm{O}_{2}$ from Conway (1988). The photoionization of $\mathrm{N}_{2}$ (into $\mathrm{N}_{2}^{+}$and $\mathrm{N}^{+}$) and $\mathrm{O}$ (into $\mathrm{O}^{+}$) are the primary source of electrons for the nonpolar ionosphere. These energetic electrons further impact with the neutral species to create more ionization, molecular dissociation, and excitation and thus to redistribute the electron energy flux. As shown in Figure 13, the photoionization of $\mathrm{O}_{2}$ has minor contributions with its largest contribution in the $0-10 \mathrm{~nm}$ range. The photoionization of $\mathrm{N}_{2}$ dominates for wavelengths shorter than $10 \mathrm{~nm}$. The photoionization into $\mathrm{N}_{2}^{+}$and $\mathrm{O}^{+}$each contribute about $50 \%$ for wavelengths longer than $10 \mathrm{~nm}$. The photoionization of $\mathrm{N}_{2}$ into $\mathrm{N}^{+}$is most important below $30 \mathrm{~nm}$. Although $\mathrm{N}_{2}^{+}$and $\mathrm{O}^{+}$are the dominant ions created, the ionosphere composition in the $\mathrm{F}$ region and higher is expected to be dominated by atomic species because the molecular ions have a much faster recombination rate (on the order of minutes) than the atomic ions (on the order of hours). One exception is in the lower E region where the longer wavelength EUV and FUV radiation is responsible for the creation of $\mathrm{O}_{2}^{+}$ and $\mathrm{NO}^{+}$molecular ions.

\subsubsection{Comparisons to Dayglow Measurements}

The XUV irradiance results from XPS are mostly important for comparisons with ionospheric responses. One comparison for the XPS Level 4 products is with the electron density, such as the total electron content (TEC) and photoelectron flux as measured by the FAST satellite. Another useful comparison is with the thermospheric dayglow results, which are sensitive to electron density. These electron-impact dayglow emissions include the Lyman-Birge-Hopfield (LBH) bands of $\mathrm{N}_{2}$ and the 135.6-nm emission of $\mathrm{O}$, which 

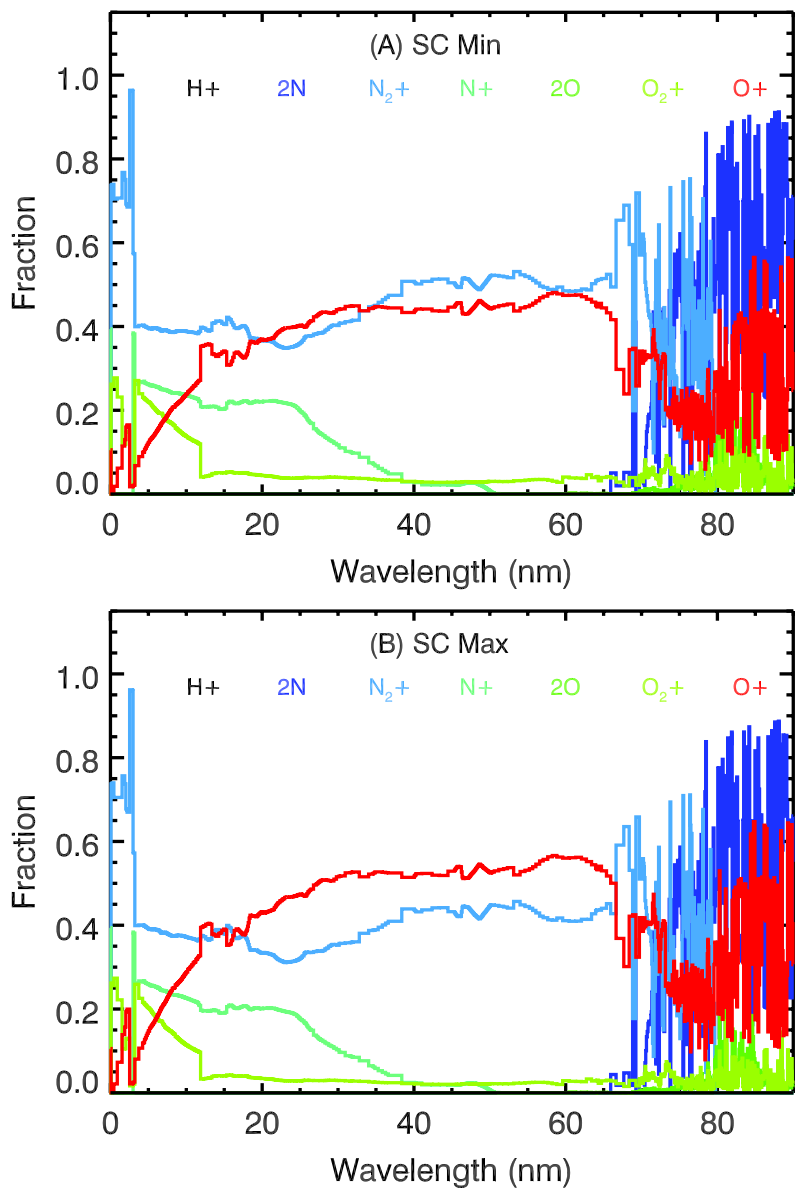

Figure 13 Fraction of atmospheric absorption divided into species from photodissociation and photoionization. The top (A) and bottom (B) panels represent the hemispheric average for the atmosphere densities at solar minimum and maximum, respectively. In both cases, the ionization of $\mathrm{O}$ and $\mathrm{N}_{2}$ dominate for wavelengths shorter than $70 \mathrm{~nm}$.

are observed by the TIMED Global UltraViolet Imager (GUVI) (Christensen et al., 2003). Because of possible auroral contributions at high latitudes, the changes in the ionosphere at lower latitudes are more valid for comparison with the solar XUV irradiance variations.

The GUVI instrument measures the concentrations of atomic oxygen and molecular nitrogen and oxygen by observing their photoelectron-excited FUV dayglow emissions (Christensen et al., 2003). Observations of $\mathrm{O}(135.6 \mathrm{~nm})$ and two intervals of $\mathrm{N}_{2} \mathrm{LBH}$ bands $(141.0-152.8$ and $167.2-181.2 \mathrm{~nm})$ on the Earth limb between about 110 and $510 \mathrm{~km}$ tangent altitude can be inverted to yield the variations of the species concentrations with altitude and latitude (Meier and Picone, 1994; Christensen et al., 2003). The two $\mathrm{N}_{2}$ bands are called LBHS (short wavelength) and LBHL (long). Meier and Picone (1994) discuss the inversion methodology, which has been upgraded as described by Christensen et al. (2003).

In addition to parameters that define the concentrations of $\mathrm{O}, \mathrm{N}_{2}$, and $\mathrm{O}_{2}$ and temperature and how they vary with altitude, three other parameters are retrieved that allow for differ- 
ences between the magnitudes of the column emission rates observed and those predicted by the forward model in the inversion algorithm. These three are called magnitude scalars of the predicted emission rates for each of the three GUVI channels. The three are redundant, in principle, if the forward model correctly represents the spectral content of the dayglow and if the relative sensitivity of the GUVI instrument is known and unchanging. For the current version of the limb database (GUVI version 6), the three independent scalars have been retained.

The magnitude of the column emission rate from the forward model is primarily determined by three factors: the adopted solar spectral irradiance, the number densities of the emitting species, and the photoelectron impact excitation cross section. Other quantities such as the photoelectron energy loss cross sections or the EUV extinction cross sections are important, but are assumed to be known from laboratory measurements. Version 6 of the GUVI limb database uses the SEE version 9 daily averaged spectral irradiances as input to the forward model of the dayglow. Meier and Picone (1994) have shown that the number densities of the species are retrieved from the altitude dependence of the dayglow layer and, in principle, are independent of the magnitude of the emission rate. Thus, the scaling factors compensate for any mismatch between the calibrated GUVI emission rates and the product of the solar spectral irradiance (as transformed through the photoelectron flux) and the cross sections. If the retrieved magnitude scalars do not have mean values of unity, there is a systematic uncertainty in the GUVI calibration, the SEE calibration, or the cross sections or a combination of them.

Recently, an intercomparison between stellar and simultaneous dayglow observations by GUVI and by the Special Sensor Ultraviolet Spectrographic Imager (SSUSI) instrument on the Defense Meteorological Satellite Program (DMSP) satellite has concluded that the GUVI responsivities require adjustment (D. Morrison, private communication, 2007). Radiances in the O I $135.6 \mathrm{~nm}$, LBHS, and LBHL channels need to be modified by $+13 \%$, $+23.9 \%$, and $-8.1 \%$, respectively. These changes have not yet been implemented in the various data sets on the GUVI Web site, but they have been incorporated in the present work. The accuracy of the in-flight responsivity calibration is estimated to be better than $15 \%$.

The GUVI limb database extends from day 60 of 2002 through day 97 of 2007 . Retrievals are not carried out when the solar zenith angle at the limb exceeds $80^{\circ}$ owing to potential errors in modeling the photoelectron flux near the terminator. This leads to gaps in the database because of the precession of the TIMED orbit in local time. More than 400 limb scans have been inverted so far. Recently, the limb-retrieval algorithm has been modified to use the SEE irradiances at their once-an-orbit cadence (SEE Level 3A data product). For optimal accuracy, we restrict the intercomparison between GUVI and SEE to those times when GUVI limb observations take place within the 3-minute interval (per orbit) of SEE measurements. The number of simultaneous events is 4914 . To reduce the spread in data points, the GUVI data were limited to solar zenith angles less than $60^{\circ}$ and low geomagnetic activity conditions $(\mathrm{Ap}<10)$.

Figure 14 shows a plot of the magnitude scalars for the three GUVI channels as a function of the integrated energy flux from $0-45 \mathrm{~nm}$ as measured by SEE. This part of the spectral irradiance is responsible for production of most of the photoelectron flux with sufficient energy to excite the FUV emissions observed by GUVI. Figure 14 shows no systematic variation in the magnitude scalars with solar activity (to within the statistical variations), thereby implying no temporal trend from 2002 (more active) to 2007 (quiet conditions). The previous version of XPS data products exhibited a systematic downward trend with solar cycle activity, so we consider the new XPS results to be significantly improved. The mean 


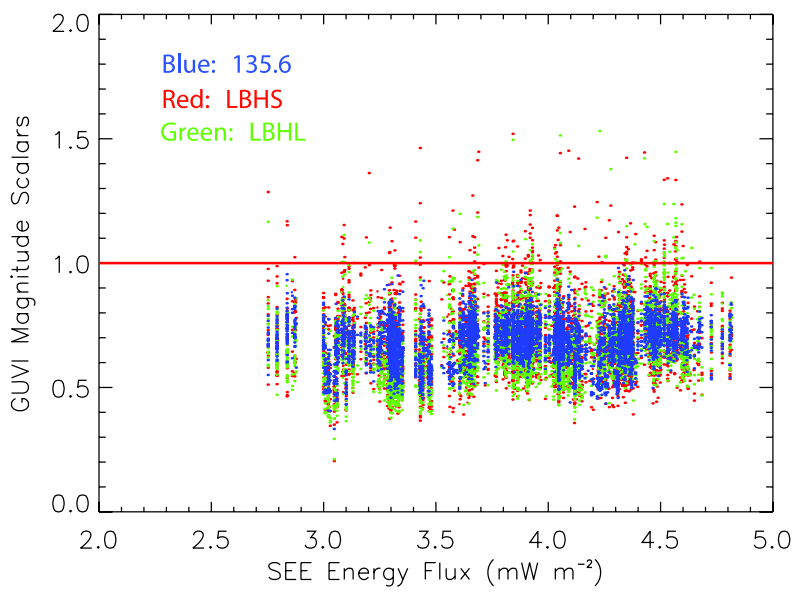

Figure 14 Comparison of new TIMED XPS Level 4 results to GUVI magnitude scalars of the $0-45 \mathrm{~nm}$ irradiance. The three GUVI results derived from the O I $135.6 \mathrm{~nm}$ (135.6), $\mathrm{N}_{2}$ LBH short (LBHS: $140-150 \mathrm{~nm}$ ), and $\mathrm{N}_{2}$ LBH long (LBHL: $165-180 \mathrm{~nm}$ ) dayglow emissions indicate a consistent offset of $0.67-0.69$ and are independent of solar activity (i.e., the trend is flat). This scalar value indicates that XPS measurements are higher than what the dayglow brightness would predict. This result suggests a systematic offset between the XPS and GUVI calibrations.

values and standard deviations for O I $135.6 \mathrm{~nm}$, LBHS, and LBHL magnitude scalars are $0.68 \pm 0.09,0.69 \pm 0.15$, and $0.66 \pm 0.15$, respectively, implying that the forward model dayglow predictions using SEE spectral irradiances are larger than observed by GUVI. The standard deviations are much larger than the differences among the means, indicating good internal consistency in the GUVI relative calibration and excitation cross sections.

Although the scalar values are within the $20-40 \%$ calibration uncertainties of both SEE and GUVI, this result does suggest a systematic offset between the XPS and GUVI calibrations. The cross section used in the limb retrievals for photoelectron impact excitation of $\mathrm{O}\left({ }^{5} \mathrm{~S}\right.$ ), the parent state of the 135.6-nm emission, is $9 \times 10^{-18} \mathrm{~cm}^{2}$ at $18 \mathrm{eV}$ (Stone and Zipf, 1974), adjusted for cascade and revised calibration standards by Meier (1991). For $\mathrm{N}_{2}\left(\mathrm{a}^{1} \Pi_{\mathrm{g}}\right)$, the parent state of the LBH bands, the total excitation cross section used is $4.3 \times 10^{-17} \mathrm{~cm}^{2}$ at $18 \mathrm{eV}$. This cross section is based on the measurement of $3 \times 10^{-17} \mathrm{~cm}^{2}$ by Ajello and Shemansky (1985) but adjusted to the current value by accounting for revised calibration standards and cascade from the $\mathrm{a}^{1} \Sigma_{\mathrm{u}}$ and $\mathrm{w}^{1} \Delta_{\mathrm{u}}$ states of $\mathrm{N}_{2}$. Johnson et al. (2005) have more recent results on the $\mathrm{N}_{2}$ cross sections by electron impact, and those results are about a factor of 2 lower than the previous ones. R.R. Meier is currently evaluating uncertainties in the cross sections as a possible source of the systematic offset between SEE and GUVI.

\subsubsection{Comparison to Photoelectron Measurements and Models}

The ionospheric photoelectron flux measurements by FAST (Carlson et al., 2001) are also consistent with the new XPS Level 4 results. Woods et al. (2003) and others have shown that photoelectrons provide a unique method to validate the solar EUV spectral irradiance. On 15 July 2002 an X3 class flare started about 20:00 UT, peaked at 20:08 UT, and ended after 20:14 UT. Photoelectrons streaming up magnetic field lines were observed on the FAST satellite during this flare event at an altitude of about $3700 \mathrm{~km}$. Prompt X-ray emissions and 

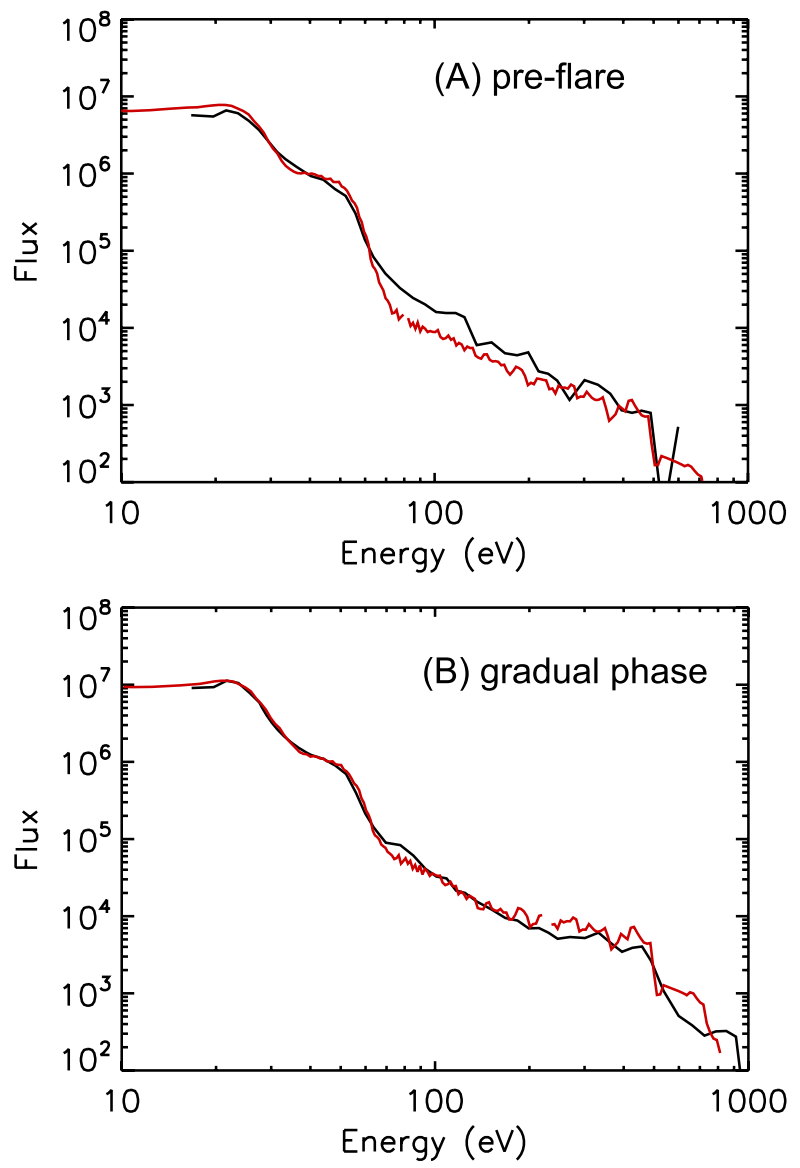

Figure 15 Comparison of FAST photoelectron measurements (black lines) to estimates from the FLIP model for the X3 flare on 15 July 2002. The FLIP model (Richards, 2002) uses as input the FISM solar EUV irradiances (Chamberlin, Woods, and Eparvier, 2007) that are based on the XPS Level 4 results. The top panel (A) shows the comparison at 20:02:55 UT as a pre-flare example, and the bottom panel (B) shows the comparison at 20:05:55 UT during the gradual phase of the flare. The main difference is near $100 \mathrm{eV}$ $(\approx 10 \mathrm{~nm}$ in the solar spectrum) for the pre-flare comparison.

intensification of the photoelectron fluxes were observed between 20:04 and 20:05 UT. Figure 15 shows observed and modeled photoelectron fluxes at 20:02:55 near the early rise of the GOES X-ray irradiance (pre-flare) and at 20:05:55 near the peak of the GOES X-ray irradiance (gradual phase of the flare). The observed photoelectron spectra have been adjusted to account for the spacecraft potential.

The modeled photoelectron spectra were obtained by using the FLIP model (Richards, 2002). The FLIP model was adapted to use solar irradiance input from FISM (Chamberlin, Woods, and Eparvier, 2007) that was updated to use the new XPS Level 4 results. This comparison suggests slightly higher solar irradiances than the new XPS results near $10 \mathrm{~nm}$ $(\approx 100 \mathrm{eV}$ for photoelectrons) for the pre-flare spectrum but good spectral agreement during the gradual (main) phase of the solar flare. The post-flare comparison shows spectral differences similar to those found in the pre-flare comparison. The good agreement above $200 \mathrm{eV}$ in the photoelectron flux indicates that the solar irradiance near $2-3 \mathrm{~nm}$ is in agreement as 
the photoelectron flux above $200 \mathrm{eV}$ is largely due to the Auger process for O near $2.2 \mathrm{~nm}$ and $\mathrm{N}_{2}$ near $3 \mathrm{~nm}$ (Richards et al., 2006). The FAST photoelectron spectra have an accuracy of only $30-40 \%$ owing to background subtraction and calibration. However, the uncertainty in the shape of the FAST photoelectron spectra is less because of the well-defined electron optics of the FAST electron detector. This comparison suggests that the spectral irradiance near $10 \mathrm{~nm}$ is not completely correct in FISM and thus for the XPS Level 4 results. Some of the differences could be the reference spectra used in the XPS Level 4 algorithm but they also could arise from temporal changes that are not captured well in the FISM estimates. Neither is really resolvable without higher spectral resolution and higher temporal cadence measurements than the XPS observations. Nonetheless, both the GUVI dayglow and FAST photoelectron measurements indicate that the lower levels of the new XPS results (versus the higher SNOE levels) are more consistent with the atmospheric measurements.

\subsubsection{Comparisons to TEC Measurements and Models}

Comparisons during large flares are very important for validating the new XPS Level 4 algorithm and results. As one example, Tsurutani et al. (2005) show that the TEC increased by about $30 \%$, or $2.5 \times 10^{13} \mathrm{e}^{-} \mathrm{cm}^{-2}$, near the subsolar point during the X17 flare on 28 October 2003. The increase of the solar irradiance shorter than $105 \mathrm{~nm}$ is expected to be the main contributor to this increase of the TEC. The XPS Level 4 product, combined with the SEE Level 3 product at wavelengths longer than $27 \mathrm{~nm}$ and up to $105 \mathrm{~nm}$, indicate an irradiance increase by a factor of 3.6 for this flare.

A rough estimate of the increase in the ionosphere is the integral over the flare period of the solar EUV spectrum that is in photon units and is weighted with the atomic ion fraction as given in Figure 13. This weighting of just the atomic ions $\left(\mathrm{N}^{+}\right.$and $\left.\mathrm{O}^{+}\right)$is more relevant because the molecular ions have a short lifetime, as mentioned earlier. This rough estimate is expected to be an upper limit if integrated over the full flare period (rise and fall) as no recombination of the atomic ions is assumed, but it is expected to be a lower limit if integrated just up to the flare peak because the molecular ions are ignored. This simple calculation yields $1.2 \times 10^{13} \mathrm{e}^{-} \mathrm{cm}^{-2}$ as a lower limit (up to flare peak) and $4.3 \times$ $10^{13} \mathrm{e}^{-} \mathrm{cm}^{-2}$ as an upper limit (over full flare) for the increase in the TEC. These rough estimates bound the measured TEC increase by about a factor of 2 in each direction.

A more realistic comparison for the TEC increase during the flare on 28 October 2003 can be made by using the measured solar irradiance changes as input to an atmospheric model. The Thermosphere, Ionosphere, and Electrodynamics General Circulation Model (TIE-GCM) (Roble et al., 1988; Richmond, Ridley, and Roble, 1992) used as input the FISM solar irradiance estimates based on XPS Level 4 results. The modeled TEC increases during the flare by as much as 11 TECU (where $1 \mathrm{TECU}=10^{12}$ electrons $\mathrm{cm}^{-2}$ ). As shown in Figure 16, the modeled TEC differences using the flare time of 11:10 UT are compared to the measured TEC differences from Figure 2 of Tsurutani et al. (2005) that used the flare time of 11:08 UT. The measured and modeled TEC changes have similar regional structure, but the measured TEC increases during the flare are generally higher than the TIE-GCM results by $2-10$ TECU. Although the measured TEC increase near the peak $(\approx 25$ TECU) is about a factor of 2 higher than the modeled TEC increase ( $\approx 11$ TECU), it is important to note that the TEC increase from the pre-flare condition is fairly small, being about $30 \%$ and $13 \%$ for the measured and modeled TEC increases, respectively. Therefore, the TEC absolute values near the peak are expected to be only about $15 \%$ more for the measurements than the TIE-GCM results.

Some of this difference between the measured and model TEC increase could be related to the uncertainty in the TEC measurements, time difference in extracting the TEC increase, 


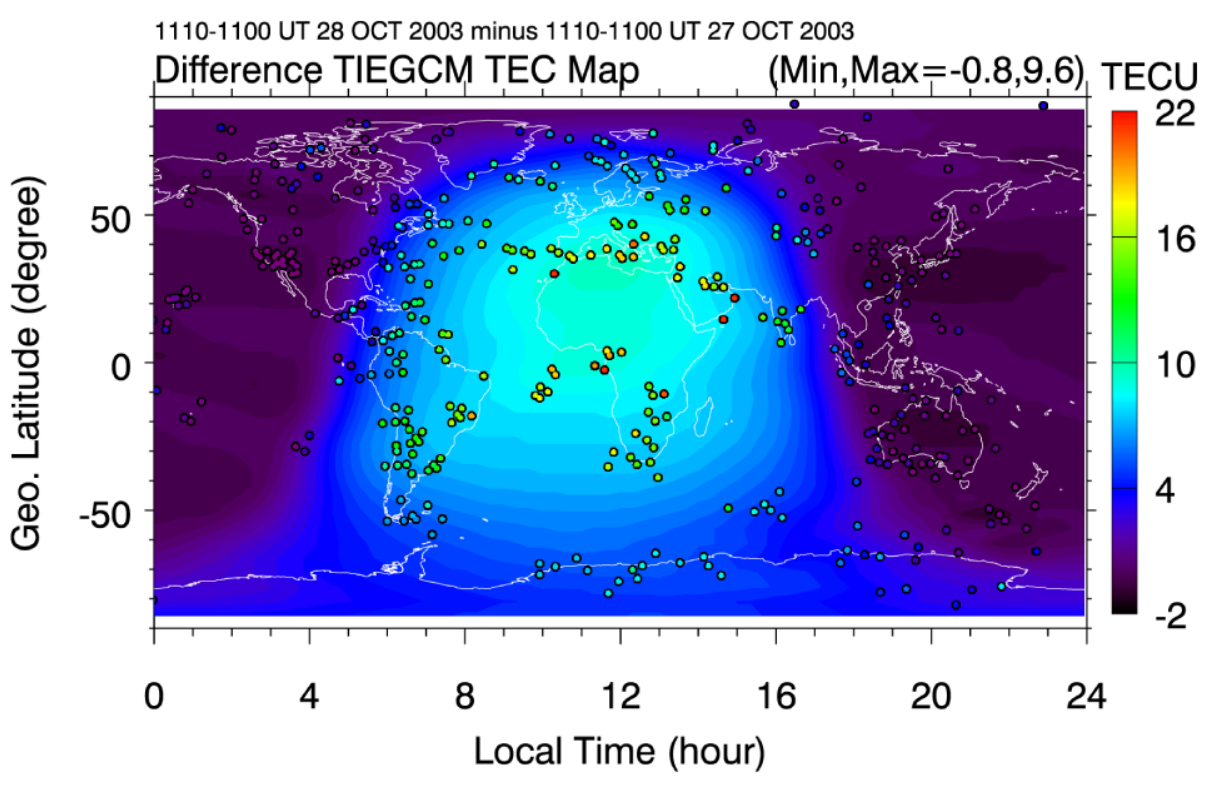

Figure 16 Comparison of measured and modeled TEC changes for the X17 flare event on 28 October 2003. The modeled TECs are from the TIE-GCM that used as input the FISM solar irradiance estimates based on XPS Level 4 results. The measured TECs (colored dots with black rings) are from Figure 2 of Tsurutani et al. (2005). Note that $1 \mathrm{TECU}$ is $10^{12}$ electrons $\mathrm{cm}^{-2}$. The measured TEC increases during the flare are generally higher than the TIE-GCM results by $2-10$ TECU.

the temporal results of the FISM solar irradiances used in TIE-GCM, the XPS Level 4 levels used to generate the FISM parameters, and/or TIE-GCM processes on time scales of flares (minutes). The GOES $0.1-0.8 \mathrm{~nm}$ irradiance rose rapidly until about 11:05 UT and then peaked at 11:10 UT. The variation of the modeled TEC difference over 10 minutes from 11:10 to 11:20 UT is less than 2 TECU at the peak; therefore, the time difference in extracting the TEC increase is not expected to be the primary cause. Because FISM is an empirical model based on XPS Level 4 results, the FISM temporal profiles are probably less suspect than the XPS Level 4 levels. A detailed comparison of the absolute values of the TEC versus TEC differences is warranted for TIE-GCM validation but out of scope for the simple comparison included here.

Previous TIE-GCM results of the TEC during this flare, when using the older version of XPS results, indicated unrealistically large TEC increases, and these earlier results concerning the atmosphere response to flares were one of the motivations for updating the XPS Level 4 algorithm. It is encouraging to see that measured and modeled atmospheric responses to flares are starting to converge with the use of the new XPS Level 4 results, but not all of the differences have been resolved.

\subsubsection{Comparisons to Dayglow Flare Modeling}

As yet another flare comparison, the dayglow response to a large flare as measured by TIMED GUVI (Strickland et al., 2007) is compared to the new XPS results. One of the GUVI results in the analysis of the $\mathrm{O}$ and $\mathrm{N}_{2}$ dayglow emissions is the integrated solar irradiance in the $1-45 \mathrm{~nm}$ range, $Q_{\mathrm{EUV}}$. Using a multiple iteration technique to fit the FUV dayglow emission increases, Strickland et al. (2007) estimate the solar irradiance in broad bands 
Table 1 Comparison of TIMED GUVI results for the X17 flare on 28 October 2003. The first three rows include the results listed in Table 2 of Strickland et al. (2007) for GUVI Rev 10219. All numbers are the solar irradiance in units of $\mathrm{mW} \mathrm{m}^{-2}$. The $27-45 \mathrm{~nm}$ irradiance has the same value for both SEE version 8 and version 9 (with the new XPS Level 4 result being part of SEE version 9). The new GUVI modeling result for the case of the solar $1-5 \mathrm{~nm}$ irradiance being $9.6 \mathrm{~mW} \mathrm{~m}^{-2}$ is listed as "Strickland-2"

\begin{tabular}{lllll}
\hline Source & $\begin{array}{l}\text { Solar } \\
1-5 \mathrm{~nm}\end{array}$ & $\begin{array}{l}\text { Solar } \\
5-27 \mathrm{~nm}\end{array}$ & $\begin{array}{l}\text { SEE } \\
27-45 \mathrm{~nm}\end{array}$ & $\begin{array}{l}\text { Total } \\
1-45 \mathrm{~nm}\end{array}$ \\
\hline Strickland & 2.0 & 7.2 & 2.8 & 12.0 \\
Strickland & 4.0 & 6.2 & 2.8 & 13.0 \\
Strickland & 6.0 & 5.3 & 2.8 & 14.1 \\
SORCE XPS L4 & 6.3 & 6.1 & 2.8 & 15.2 \\
Strickland-2 & 9.6 & 3.5 & 2.8 & 15.9 \\
TIMED XPS L4 & 9.6 & 4.7 & 2.8 & 17.1 \\
\hline
\end{tabular}

Figure 17 Comparison of new XPS Level 4 results to the GUVI estimated spectrum in the $1-45 \mathrm{~nm}$ range. The technique of Strickland et al. (2007) is used to produce the GUVI estimated spectrum for two cases: 9.6 and $6.0 \mathrm{~mW} \mathrm{~m}^{-2}$ for the $1-5 \mathrm{~nm}$ range. The ratios of the GUVI result for the $9.6 \mathrm{~mW} \mathrm{~m}^{-2}$ case to other results are indicated in the bands scaled in the GUVI analysis, being $5-10,10-15$, $15-20$, and $20-27 \mathrm{~nm}$. The other GUVI analysis band at $27-45 \mathrm{~nm}$ is given by the measured TIMED EGS spectrum

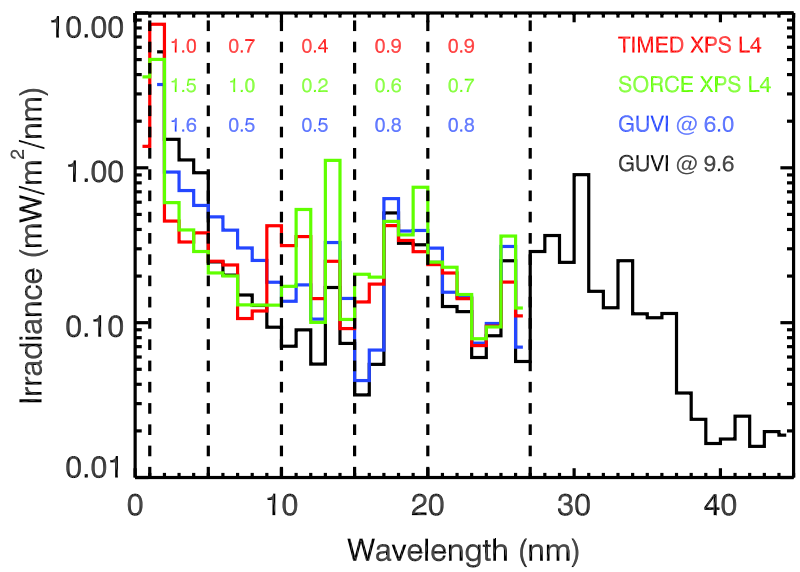

of $1-5,5-10,10-15,15-20$, and $20-27 \mathrm{~nm}$ and use measurements elsewhere. Their result for the X17 flare on 28 October 2003 indicated an overestimate of the solar irradiance near the flare peak for the TIMED SEE version 8 data, but the new XPS Level 4 results are now much more consistent with the GUVI analysis results, as indicated in Table 1 and Figure 17. Strickland et al. (2007) had roughly estimated the $1-5 \mathrm{~nm}$ irradiance and listed a few options for that band and then fit the bands in the 5-27 nm range. The new TIMED XPS Level 4 results indicate a higher flare irradiance for the $1-5 \mathrm{~nm}$ band $\left(9.6 \mathrm{~mW} \mathrm{~m}^{-2}\right.$ versus their original estimates of 2,4 , and $6 \mathrm{~mW} \mathrm{~m}^{-2}$ ). Their new result in the $5-27 \mathrm{~nm}$ range (listed as Strickland-2 in Table 1) is consistent with the new TIMED XPS Level 4 result with the total $1-45 \mathrm{~nm}$ irradiance results having a difference of only $7 \%$. There are some larger differences as shown in Figure 17. The TIMED XPS result is more consistent with the GUVI $9.6 \mathrm{~mW} \mathrm{~m}^{-2}$ case, whereas the SORCE XPS result is more consistent with the GUVI $6.0 \mathrm{~mW} \mathrm{~m}^{-2}$ case. Some of this difference may be due to the two different XPS instruments, but some of the difference is due to the actual time of the different measurements during the flare phase. The largest spectral differences are in the $5-15 \mathrm{~nm}$ range whereby the XPS results have much larger irradiances in the 10-15 range than the GUVI results. The FAST photoelectron measurements suggest that the XPS results are likely to be more correct. This difference is most likely related to the different reference spectra used in both 
XPS and GUVI analyses. A more detailed analysis for additional flares is desirable, but this comparison is encouraging as the GUVI results and XPS solar irradiance measurements are in good agreement, for the first time, for this flare example. Their differences are within the $40 \%$ uncertainty of the XPS Level 4 irradiances, except for the $10-15 \mathrm{~nm}$ range.

\section{Summary}

The XPS responsivities have been revised by combining the best of three different calibrations and are an improvement for the new XPS irradiance data products. The primary change in the new XPS irradiance level is higher irradiances in the $17-27 \mathrm{~nm}$ range by about a factor of 1.5. These new XPS results are still lower than the SNOE results by a factor of 1.5. The new XPS results are considered more accurate than SNOE results because the XPS results are more consistent with the thermospheric dayglow measurements by TIMED GUVI and the ionospheric photoelectron flux measurements by FAST.

A new algorithm for processing the broadband measurements from the XPS has been developed that uses three components from the CHIANTI spectral model. There are two daily components that are used to match the daily minimum signal from the XPS $0.1-7 \mathrm{~nm}$ channel and one flare component that is used to match the signal above the daily minimum signal. The daily components include a solar cycle minimum irradiance component and an active region component. The solar cycle minimum irradiance component consists of the average of the quiet Sun DEM and coronal hole DEM options in the CHIANTI model, and the active region component is the active region DEM option in the CHIANTI model. The scale factor for the solar cycle minimum irradiance component is set to be 1.0 , and the scale factor for the active region component is set to match the daily minimum signal. The active region scale factor ranges from 0 near solar minimum to about 0.04 near solar maximum, and this scale factor shows solar rotation and solar cycle changes that are representative of the fractional area of active regions on the solar disk. The flare component uses an isothermal option in the CHIANTI model, and the flare temperature is determined from the ratio of the GOES $0.05-0.4 \mathrm{~nm}$ band to the $0.1-0.8 \mathrm{~nm}$ band (Garcia, 1994). The flare temperature is usually near $10 \mathrm{MK}$, so most of the flare spectra are dominated by a peak in the irradiance near $2 \mathrm{~nm}$. Although flares are known not to be isothermal, this approach is deemed acceptable because the XPS results have similar XUV spectra from the full DEM analysis of flares by Rodgers et al. (2006) and are also consistent with the detailed analysis for the flare DEMs by Chifor et al. (2007).

The XPS Level 4 data product includes the model scale factors, flare temperatures, and adjusted model spectra in the $0.1-40 \mathrm{~nm}$ range in $0.1-\mathrm{nm}$ intervals, and results are given for each measurement made by the XPS Ti-coated photometers $(0.1-7 \mathrm{~nm}$ bandpass). The daily SORCE XPS Level 4 data products are also merged into a mission data file, both in daily averages and in 5-minute averages. The uncertainty of the XPS Level 4 products is estimated to be about $40 \%$, which is considered a significant improvement over historical results that sometimes included differences by factors of $2-3$.

The motivation for revising the XPS processing algorithm was to provide more realistic flare results from the XPS broadband measurements. The previous XPS flare results indicated XUV variations that were factors of 2 or more than the atmospheric response to the flares. The new XPS algorithm is producing results that are consistent with the daily averages of the XPS broadband irradiances, the TIMED SEE EGS spectral measurements in the $27-40 \mathrm{~nm}$ range, and reduced flare variations as compared to the previous XPS version. The flare variations are now reduced by about a factor of 4 in the $7-17 \mathrm{~nm}$ range and a 
factor of 2 in the $0-7 \mathrm{~nm}$ range. The new flare variations are slightly larger by about $20 \%$ in the 17-27 $\mathrm{nm}$ range than the previous XPS version and are also consistent with the spectral measurements by EGS in the $27-40 \mathrm{~nm}$ range. The primary difference between the new XPS results and EGS is for the He II $30.4 \mathrm{~nm}$ optically thick emission, but the CHIANTI spectral model is known to have difficulty handling optically thick emissions.

These improvements for the flare irradiances are also realized in comparisons with the ionospheric enhancements measured during flares. Using the XPS-based FISM estimates of the solar irradiance, the FLIP model of the photoelectron flux during the flare on 15 July 2002 is in good agreement with the FAST photoelectron measurements. As another example, the measured TEC increased from the X17 flare on 28 October 2003 by about $30 \%$ or 25 TECU near the subsolar point (Tsurutani et al., 2005), and detailed modeling of the ionospheric response to this flare by the TIE-GCM model, which uses solar irradiance input based on the XPS Level 4 results, indicates a similar, but smaller, increase of about 11 TECU near the subsolar point ( $\approx 12$ hours local time). Furthermore, the comparison of these new XUV irradiance results are more consistent, for the first time, with day-to-day changes and flare responses in the FUV dayglow as measured by TIMED GUVI.

Although there is significant improvement in the XPS irradiances data set with its new Level 4 algorithm, especially for flares, it is important to note that there remain some differences in the irradiance levels. The GUVI dayglow results, as shown in Figures 14 and 17, suggest that the XPS irradiances are too high by about $30 \%$ over the full XUV range or about $60 \%$ too high in the narrower $10-15 \mathrm{~nm}$ range. Of course, some of these differences can be attributed to calibration uncertainties for both GUVI and XPS and also may be related to uncertainties in the dayglow cross sections used in GUVI processing. In contrast, the comparison of the measured and modeled TEC changes from a large flare suggests that the XPS irradiances are too low by about $20 \%$, and the FAST photoelectron comparison suggests that the XPS irradiances are too low by a factor of about 2 at wavelengths near $10 \mathrm{~nm}$. The HEUVAC model comparison, based partially on earlier photoelectron measurements, also suggests that the XPS irradiances are too low in the 5-15 nm range. These atmospheric comparisons, both for daily averaged and flare events, conflict in suggesting higher and lower XUV irradiance values; the largest differences appear at wavelengths near $10 \mathrm{~nm}$.

These conflicting conclusions may not be resolved until higher spectral resolution and higher accuracy measurements of the solar XUV irradiance become available. The next opportunity is by the SDO EVE. An SDO EVE prototype will fly on a sounding rocket in April 2008 for a solar cycle minimum measurement and under-flight calibration for TIMED SEE and SORCE XPS, and the SDO is scheduled for launch in December 2008. The SDO EVE will measure the solar radiation from 0.1 to $105 \mathrm{~nm}$ with $0.1-\mathrm{nm}$ spectral resolution at wavelengths longer than $5 \mathrm{~nm}$. In addition, the ESA Solar Auto-Calibrating EUV Spectrometers (SOL-ACES) (Wienhold et al., 2000) is planning to make new solar EUV irradiance measurements in 2008-2010 from the International Space Station. The SOL-ACES will measure the solar radiation from 17 to $220 \mathrm{~nm}$ with a spectral resolution from 0.3 to $2 \mathrm{~nm}$ with four grazing incidence planar grating spectrometers.

These new XPS Level 4 data products are applicable for inclusion in solar irradiance spectral models and for a variety of space weather research and applications. The latest version of FISM already includes these new XPS results (Chamberlin, Woods, and Eparvier, 2007). More detailed studies of the thermosphere and ionosphere responses to solar variations, on time scales both related to flare events and over the solar cycle, are needed to realize the full impact of the solar XUV irradiance on temperature and composition changes in the upper atmosphere. The improved solar XUV irradiances are then important improvements for space weather operations and forecasts of the ever-changing ionosphere and thermosphere. 
These updated XPS results are considered to be more accurate and more representative of the solar spectral variations, even during flares, but they are simply models of the solar spectrum based on broadband measurements by XPS. Many additional questions remain. How well does the CHIANTI spectral model represent the true solar spectra in the XUV range? The CHIANTI model has recently been updated with many additional XUV emissions, but what emission lines are still missing in the model? How well can a combination of daily and flare components represent the true solar spectra? Are there additional components needed? The future measurements by SDO EVE with 0.1-nm spectral resolution will surely address these outstanding concerns about the solar XUV irradiance and its spectral variations. The SDO satellite is expected to launch in late 2008, and the SDO observations by EVE and also the Atmospheric Imaging Assembly instrument will initiate a new validation effort of these XPS results and will likely lead to improvements for CHIANTI and other spectral models of the Sun.

Acknowledgements This research was supported by NASA Contract No. NAS5-97045 and NASA Grant No. NNX07AB68G to the University of Colorado and NASA Grant No. NNX07AN03G to George Mason University. In addition, portions of the work were performed at the Jet Propulsion Laboratory, California Institute of Technology, under contract with NASA. We thank Brian Templeman and Don Woodraska for their support of data processing of the SORCE and TIMED XPS data and Vanessa George for her assistance with this manuscript. The CHIANTI spectral model is a collaborative project involving the NRL (USA), RAL (UK), MSSL (UK), the Universities of Florence (Italy) and Cambridge (UK), and George Mason University (USA). The SORCE data are available from http://lasp.colorado.edu/sorce/. The TIMED SEE data are available from http://lasp.colorado.edu/see/.

Open Access This article is distributed under the terms of the Creative Commons Attribution Noncommercial License which permits any noncommercial use, distribution, and reproduction in any medium, provided the original author(s) and source are credited.

\section{References}

Ajello, J.M., Shemansky, D.E.: 1985, J. Geophys. Res. 90, 9845.

Aschwanden, M.J.: 2004, Physics of the Solar Corona, Springer, Berlin.

Aschwanden, M.J.: 2007, Astrophys. J. 661, 1242.

Bailey, S.M., Woods, T.N., Barth, C.A., Solomon, S.C., Canfield, L.R., Korde, R.: 2000, J. Geophys. Res. 105, 27179.

Bailey, S.M., Woods, T.N., Barth, C.A., Solomon, S.C., Canfield, L.R., Korde, R.: 2001, J. Geophys. Res. 106, 15791 .

Brekke, P., Rottman, G.J., Fontenla, J., Judge, P.G.: 1996, Astrophys. J. 468, 418.

Carlson, C.W., McFadden, J.P., Turin, P., Curtis, D.W., Magoncelli, A.: 2001, Space Sci. Rev. 98, 33.

Chamberlin, P.C., Woods, T.N., Eparvier, F.G.: 2007, Space Weather 5, S07005.

Chifor, C., Del Zanna, G., Mason, H.E., Sylwester, J., Sylwester, B., Phillips, K.J.H.: 2007, Astron. Astrophys. 462, 323.

Christensen, A.B., Paxton, L.J., Avery, S., Craven, J., Crowley, G., Humm, D.C., et al.: 2003, J. Geophys. Res. 108, 1451.

Conway, R.R.: 1988, Photoabsorption and Photoionization Cross Sections of $\mathrm{O}, \mathrm{O}_{2}$, and $\mathrm{N}_{2}$ for Photoelectron Production Calculations: A Compilation of Recent Laboratory Measurements NRL-MR-6155, Naval Res. Lab., Washington, D.C.

Dere, K.P., Landi, E., Mason, H.E., Monsignori Fossi, B.C., Young, P.R.: 1997, Astron. Astrophys. Suppl. 125, 149.

Garcia, H.A.: 1994, Solar Phys. 154, 275.

Garcia, H.: 2000, Astrophys. J. Suppl. 127, 189.

Hinteregger, H.E., Fukui, K., Gilson, G.R.: 1981, Geophys. Res. Lett. 8, 1147.

Johnson, P.V., Malone, C.P., Kanik, I., Tran, K., Khakoo, M.A.: 2005, J. Geophys. Res. 110, A11311.

Judge, D.L., McMullin, D.R., Ogawa, H.S., Hovestadt, D., Klecker, B., Hilchenbach, M., et al.: 1998, Solar Phys. 177, 161. 
Kintner, P.M., Ledvina, B.M., de Paula, E.R.: 2007, Space Weather 5, S09003.

Landi, E., Del Zanna, G., Young, P.R., Dere, K.P., Mason, H.E., Landini, M.: 2006, Astrophys. J. Suppl. 162, 261.

Lanzerotti, L.J.: 2001, In: Song, P., Singer, H.J., Siscoe, G.L. (eds.) Space Weather, AGU Monograph 125, AGU, Washington

Lean, J.: 1987, J. Geophys. Res. 92, 839.

Lean, J.: 1991, Rev. Geophys. 29, 505.

Liedahl, D.A., Osterheld, A.L., Goldstein, W.H.: 1995, Astrophys. J. 438, L115.

Marcos, F.A., Wise, J.O., Kendra, M.J., Grossbard, N.J., Bowman, B.R.: 2005, Geophys. Res. Lett. 32, L04103.

Meier, R.R.: 1991, Space Sci. Rev. 58, 1.

Meier, R.R., Picone, J.M.: 1994, J. Geophys. Res. 99, 6307.

Meier, R.R., Warren, H.P., Nicholas, A.C., Bishop, J., Huba, J.D., Drob, D.P., et al.: 2002, Geophys. Res. Lett. 29, $99-1$.

Mewe, R., Gronenschild, E.H.B.M.: 1981, Astron. Astrophys. Suppl. 45, 11.

Mewe, R., Gronenschild, E.H.B.M., van den Oord, G.H.J.: 1985, Astron. Astrophys. Suppl. $62,197$.

Mewe, R., Lemen, J.R., van den Oord, G.H.J.: 1986, Astron. Astrophys. 65, 511.

Neupert, W.M.: 1968, Astrophys. J. 153, L59.

Pap, J.M., Fröhlich, C., Hudson, H.S., Solanki, S.K. (eds.): 1994, The Sun as a Variable Star: Solar and Stellar Irradiance Variations, Cambridge University Press, Cambridge.

Picone, J.M., Hedin, A.E., Drob, D.P., Aikin, A.C.: 2003, J. Geophys. Res. 107, 1468.

Qian, L., Roble, R.G., Solomon, S.C., Kane, T.J.: 2006, Geophys. Res. Lett. 33, L23705.

Richards, P.G.: 2002, J. Geophys. Res. 107, 10.

Richards, P.G., Fennelly, J.A., Torr, D.G.: 1994, J. Geophys. Res. 99, 8981.

Richards, P.G., Woods, T.N., Peterson, W.K.: 2006, Adv. Space Res. 37, 315.

Richmond, A.D., Ridley, E.C., Roble, R.G.: 1992, Geophys. Res. Lett. 19, 601.

Roble, R.G., Ridley, E.C., Richmond, A.D., Dickinson, R.E.: 1988, Geophys. Res. Lett. 15, 1525.

Rodgers, E.M., Bailey, S.M., Warren, H.P., Woods, T.N., Eparvier, F.G.: 2006, J. Geophys. Res. 111, A10S13.

Rottman, G.J.: 1987, In: Foukal, P. (ed.) Solar Radiative Output Variation, Cambridge Research and Instrumentation, Boulder, 71.

Rottman, G.: 2005, Solar Phys. 230, 7.

Smith, R.K., Brickhouse, N.S., Liedahl, D.A., Raymond, J.C.: 2001, Astrophys. J. 556, L91.

Solomon, S.C., Qian, L.: 2005, J. Geophys. Res. 110, A10306.

Stone, E.J., Zipf, E.C.: 1974, J. Chem. Phys. 60, 4237.

Strickland, D.J., Lean, J.L., Daniell Jr., R.E., Knight, H.K., Woo, W.K., Meier, R.R., et al.: 2007, J. Geophys. Res. 112, A06313.

Sutton, E.K., Forbes, J.M., Nerem, R.S., Woods, T.N.: 2006, Geophys. Res. Lett. 33, L22101.

Tobiska, W.K.: 1993, J. Geophys. Res. 98, 18879.

Tobiska, W.K., Woods, T.N., Eparvier, F.G., Viereck, R., Floyd, L., Bouwer, D., Rottman, G.J., White, O.R.: 2000, J. Atmos. Solar-Terr. Phys. 62, 1233.

Tsurutani, B.T., Judge, D.L., Guarnieri, F.L., Gangopadhyay, P., Jones, A.R., Nuttall, J., et al.: 2005, Geophys. Res. Lett. 32, L03S09.

Warren, H.P., Mariska, J.T., Lean, J.: 2001, J. Geophys. Res. 106, 15745.

Wienhold, F.G., Anders, J., Galuska, B., Klocke, U., Knothe, M., Riedel, W.J., Schmidtke, G., Singler, R., Ulmer, U., Wolf, H.: 2000, Phys. Chem. Earth 25, 473.

White, O.R. (ed.): 1977, The Solar Output and Its Variation, Colorado Assoc. Univ. Press, Boulder.

Woods, T.N., Rottman, G.: 2005, Solar Phys. 230, 375.

Woods, T.N., Rottman, G., Vest, R.: 2005, Solar Phys. 230, 345.

Woods, T.N., Kopp, G., Chamberlin, P.C.: 2006, J. Geophys. Res. 111, A10S14.

Woods, T.N., Lean, J.L., Eparvier, F.G.: 2006, In: Gopalswamy, N., Bhattacharyya, A. (eds.) Proc. International Living With a Star Workshop, Quest Publications, Mumbai, 145.

Woods, T., Rodgers, E., Bailey, S., Eparvier, F., Ucker, G.: 1999, In: Larar, A.M. (ed.) Optical Spectroscopic Techniques and Instrumentation for Atmospheric and Space Research III, Proc. SPIE 3756, 255.

Woods, T.N., Tobiska, W.K., Rottman, G.J., Worden, J.R.: 2000, J. Geophys. Res. 105, 27195.

Woods, T.N., Bailey, S.M., Peterson, W.K., Warren, H.P., Solomon, S.C., Eparvier, F.G., Garcia, H., Carlson, C.W., McFadden, J.P.: 2003, Space Weather 1, 1001.

Woods, T.N., Acton, L.W., Bailey, S., Eparvier, F., Garcia, H., Judge, D., et al.: 2004a, In: Pap, J., Fox, P., Fröhlich, C., Hudson, H.S., Kuhn, J., McCormack, J., North, G., Sprig, W., Wu, S.T. (eds.) Solar Variability and Its Effect on Climate, Geophys. Monograph Series 141, AGU, Washington, 127. 
Woods, T.N., Eparvier, F.G., Fontenla, J., Harder, J., Kopp, G., McClintock, W.E., Rottman, G., Smiley, B., Snow, M.: 2004b, Geophys. Res. Lett. 31, L10802.

Woods, T.N., Eparvier, F.G., Bailey, S.M., Chamberlin, P.C., Lean, J., Rottman, G.J., Solomon, S.C., Tobiska, W.K., Woodraska, D.L.: 2005, J. Geophys. Res. 110, A01312.

Worden, J.R., White, O.R., Woods, T.N.: 1998, Astrophys. J. 496, 998.

Worden, J., Woods, T.N., Neupert, W.M., Delaboundiniere, J.P.: 1999, Astrophys. J. 511, 965. 